\title{
Asymptotic behavior of mild solutions for a class of abstract nonlinear difference equations of convolution type
}

\author{
Valentin Keyantuo', Carlos Lizama2* (1D, Silvia Rueda² and Mahamadi Warma'
}

\author{
"Correspondence: \\ carlos.lizama@usach.cl \\ 2 Departamento de Matemática y \\ Ciencia de la Computación, \\ Facultad de Ciencias, Universidad de \\ Santiago de Chile, Santiago, Chile \\ Full list of author information is \\ available at the end of the article
}

\begin{abstract}
We prove the existence and uniqueness of a weighted pseudo asymptotically mild solution to the following class of abstract semilinear difference equations:

$$
u(n+1)=A \sum_{k=-\infty}^{n} a(n-k) u(k+1)+\sum_{k=-\infty}^{n} b(n-k) f(k, u(k)), \quad n \in \mathbb{Z}
$$

where $A$ is the generator of a resolvent sequence $\{S(n)\}_{n \in \mathbb{N}_{0}}$ of bounded and linear operators defined in a Banach space $X$, the sequences $a, b$ are complex-valued, and $f \in l^{1}(\mathbb{Z} \times X, X)$.
\end{abstract}

MSC: 39A14; 45D05; 35B40; 47D06

Keywords: Weighted pseudo asymptotically mild solutions; Abstract difference equations; Resolvent sequences of operators

\section{Introduction}

Nowadays, continuous-time models for systems arising in science, engineering business, and social sciences have been widely studied by many researchers. However, it is sometimes necessary to discretize the continuous models for practical purposes. In some cases, the natural models can be discrete; in addition, the results of the discrete time models are more accurate and convenient than continuous models to describe phenomena such as infectious diseases, traffic dynamics, radioactivity, among others. Discrete models should preserve as much as possible the qualitative properties of the corresponding continuous time models $[2,7,8,12,23,24]$.

A systematic study of difference equations may be traced to papers by different authors $[11,15,17,26,30,32,33]$. In [33] Xia established some sufficient criteria for the existence and uniqueness of the discrete weighted pseudo almost automorphic solutions to the Volterra difference equations of convolution type as well as to nonautonomous semilinear difference equations. Elaydi obtained some of the fundamental results on the stability and asymptotic behavior of linear Volterra difference equations using the method of $Z$-transform for equations of convolution type in [17].

(c) The Author(s) 2019. This article is distributed under the terms of the Creative Commons Attribution 4.0 International License (http://creativecommons.org/licenses/by/4.0/), which permits unrestricted use, distribution, and reproduction in any medium, provided you give appropriate credit to the original author(s) and the source, provide a link to the Creative Commons license, and indicate if changes were made. 
The problem of the existence and uniqueness of solutions for differential-difference equations, that is, continuous in space and discrete in time, has been the subject of increasing interest in the last years. They are frequently analyzed in an abstract setting of Banach space. For instance, the study of maximal regularity for discrete time abstract Cauchy problems in Banach spaces has been addressed in [4, 9, 10, 21, 22, 27]. Blunck established sufficient conditions for maximal regularity of an operator $T$ on $L_{p}$ in [10]. Kemmochi [21, 22] considered a discrete Cauchy problem in a Banach space $X$, and showed that continuous maximal regularity implies discrete maximal regularity for general $\theta$-methods in the case of UMD spaces. In [27] Lizama and Murillo presented a method based on operatorvalued Fourier multipliers to characterize the existence and uniqueness of $l_{p}$-solutions for some discrete time fractional models.

Recently, in $[1,5,34]$ the authors considered the nonlinear difference equations

$$
\Delta^{\alpha} u(n)=A u(n+1)+f(n), \quad n \in \mathbb{Z},
$$

for $0<\alpha \leq 1$, where $A$ is the generator of a resolvent sequence contained in the space of all bounded linear operators defined in a Banach space. Here $\Delta^{\alpha}$ denotes fractional difference in the Weyl-like sense (see Definition 3.16), and $f$ satisfies Lipschitz conditions of global and local type. The authors studied existence, uniqueness of discrete weighted pseudo $\mathrm{S}$-asymptotically $\omega$-periodic mild solutions and asymptotic behavior for nonlinear fractional difference equations.

The most interesting feature of the works cited above is that the authors studied the fractional modeling of partial differential equations that can be modeled in discrete time as well as in continuous space. It is worthwhile to observe that if $\alpha=\tau=\theta=1$, then the discrete Cauchy problem in $X$,

$$
\left\{\begin{array}{l}
\frac{u(n+1)-u(n)}{\tau}=A u(n+\theta)+f(n+\theta), \quad n \in \mathbb{N}, \\
u(0)=0,
\end{array}\right.
$$

where $\tau>0$ is the time step, $\theta \in[0,1]$ is a fixed parameter, $u=u(n)$ is an unknown $X$ valued sequence, and $f=f(n)$ is a given one, which was studied in [21] can be represented abstractly by (1.1).

Our motivation in this paper arises from the observation that equations of type (1.1) are subsumed under the general framework of nonlinear fractional difference equations of the type

$$
u(n+1)=A \sum_{k=-\infty}^{n} a(n-k) u(k+1)+\sum_{k=-\infty}^{n} b(n-k) f(k, u(k)), \quad n \in \mathbb{Z},
$$

where $a(n), b(n)$ are appropriately chosen sequences. Equation (1.2) corresponds to the special case where $a(n)=b(n)=k^{\alpha}(n)$, with

$$
k^{\alpha}(n)=\frac{\Gamma(n+\alpha)}{\Gamma(\alpha) \Gamma(n+1)}, \quad n \in \mathbb{N}_{0} .
$$

These coefficients appear in the definition of the discrete fractional Laplacian which has been the subject of many studies in recent years (see, e.g., [26] and the references therein). Example 3.14 will provide more details on this class of equations. 
Linear and nonlinear systems of differential-difference equations of Volterra type are often used to model biological populations, see [13, 16, 29]. The theory of linear Volterra difference equations of both convolution and nonconvolution types has been studied by Elaydi, Gronek, and Schmeidel in [17, 20]; the second author proved the existence of bounded solutions via Darbo's fixed point theorem using a measure of noncompactness in the space of bounded sequences.

However, the vast majority of research works related to the class of nonlinear discrete time evolution equations (1.3) are focused either in finite dimensional cases or are restricted to the case of a bounded operator $A$. Moreover, the problem of existence and uniqueness of weighted pseudo asymptotically mild solutions to (1.3) appears not to have been considered in the literature.

In this paper we have successfully solved this problem by means of an operator theoretic approach. In this way, one of our main contributions in this paper is that we propose a new definition of discrete resolvent family $\{S(n)\}_{n \in \mathbb{N}_{0}} \subset \mathcal{B}(X)$ generated by the operator $A$ in order to represent the solution of (1.3). This new concept improves [1, 34, Definition 3.1] and [5, Definition 2.11] in the special case of (1.1).

The paper is organized as follows: Sect. 2 is devoted to some notations and to recalling preliminary results. In Sect. 3, we consider two complex-valued sequences $a, b$, and under certain conditions on $f: \mathbb{Z} \rightarrow X$ and the resolvent family generated by the operator $A$, we show that

$$
u(n+1)=\sum_{k=-\infty}^{n} S(n-k) f(k)
$$

is a strong solution of the linear version of (1.3). More precisely, it is a solution of the nonhomogeneous equation in which $f$ does not depend on $u$. The operator-valued sequence $\{S(n)\}_{n \in \mathbb{N}_{0}}$ is the discrete resolvent family associated with the system (see Definition 3.2).

Another relevant result of our work is that we give necessary conditions in terms of generators of $C_{0}$-semigroups in order to prove the existence and summability of a discrete resolvent family (Theorem 3.9). It should be noted that we give an explicit form of the family involving the well-known Poisson distribution in the spirit of the work [26]. This is necessary in order to establish reasonable conditions for a new result on the existence of strong solutions for our problem, see Theorem 3.13. Additionally, we will prove that in case $a(n)=b(n), n \in \mathbb{Z}_{+}$, then the sequence of operators $S(n)$ have the following interesting representation:

$$
S(n) x=\left[\sum_{j=0}^{n-1} \phi_{j}(n)(A R(A))^{j}\right] R^{2}(A) x, \quad n \geq 2,
$$

$S(0) x=a(0) R(A) x, S(1) x=a(1) R^{2}(A) x$, where $R(A):=(1-a(0) A)^{-1}, \phi_{0}(n)=a(n), \phi_{1}(n)=$ $\sum_{k=1}^{n-1} a(n-k) a(k)$, and the sequence $\left(\phi_{j}(\cdot)\right)$ is defined recursively by

$$
\phi_{j}(n)=\sum_{k=j}^{n-1} a(n-k) \phi_{j-1}(k), \quad j \geq 2, n \in \mathbb{Z}_{+} .
$$

In Sect. 4, we present results related to the asymptotic behavior and weighted pseudo Sasymptotic $\omega$-periodic mild solutions to (1.3). We use as the main tools various fixed point 
theorems, namely the Banach fixed point theorem, Matkowski's fixed point theorem, and the Leray-Schauder alternative theorem in combination with the useful Lemma 2.1 from [34] about compactness in the vector-valued sequence space, in which we seek solutions. In the main result of Sect. 4, we suppose that $A$ is the generator of a summable discrete resolvent family $\{S(n)\}_{n \in \mathbb{N}_{0}} \subset \mathcal{B}(X), f$ satisfies a $\theta$-Lipschitz condition, and using the LeraySchauder alternative theorem, we show that there exist a sequence $(h(n))_{n \in \mathbb{Z}}$ and a mild solution $u$ of (1.3) such that $u(n)=o(h(n))$, where the positive sequence $h: \mathbb{Z} \rightarrow \mathbb{R}^{+}$satisfies appropriate convergence properties. The precise description of this result is in the context of Theorem 4.6. Finally, in the last section, we include a constructive example to illustrate the relevance and feasibility of the given hypotheses.

\section{Preliminaries}

Let $(X,\|\cdot\|)$ be a Banach space. We denote the linear space consisting of all vector-valued sequences $f: \mathbb{Z}_{+} \rightarrow X$ by $s\left(\mathbb{Z}_{+}, X\right)$, where $\mathbb{Z}_{+}=\mathbb{N} \cup\{0\}$. Note that we also use the notation $\mathbb{N}_{0}$ for the same purpose. We also use $\mathbb{Z}^{+} \backslash\{0\}$ or $\mathbb{Z}_{+} \backslash\{0\}$ for the natural numbers at several places. Let $a: \mathbb{Z}_{+} \rightarrow \mathbb{C}$ be given. If $\sum_{k=0}^{\infty}|a(k)|<\infty$, then we say that $a$ is a summable sequence. We introduce some notation on the vector-valued spaces used in the sequel.

(i) Given two Banach spaces $X$ and $Y$, we denote by $\mathcal{B}(X, Y)$ the Banach space of all bounded linear operators from $X$ to $Y$. The space $\mathcal{B}(X, Y)$ will be endowed with the operator topology or the strong operator topology depending on the context. In case $X=Y$, we write simply $\mathcal{B}(X)$.

(ii) The Sobolev space $W^{l, p}\left(\mathbb{R}_{+}\right)$of all functions $f \in L^{p}\left(\mathbb{R}_{+}\right)$whose derivatives in the sense of distributions up to order $l$ belong to $L^{p}\left(\mathbb{R}_{+}\right)$.

(iii) $l^{\infty}(\mathbb{Z}, X):=\left\{f: \mathbb{Z} \rightarrow X:\|f\|_{\infty}:=\sup _{n \in \mathbb{Z}}\|f(n)\|<\infty\right\}$.

(iv) $l_{\rho}^{p}(\mathbb{Z}, X):=\left\{f: \mathbb{Z} \rightarrow X:\|f\|_{l_{\rho}^{p}}:=\sum_{n=-\infty}^{\infty}\|f(n)\|^{p} \rho(n)<\infty\right\}$, where $\rho: \mathbb{Z} \rightarrow(0, \infty)$ is a positive sequence.

(v) $C_{0}(\mathbb{Z}, X):=\left\{f \in l^{\infty}(\mathbb{Z}, X): \lim _{n \rightarrow \infty}\|f(n)\|=0\right\}$.

(vi) $C_{\omega}(\mathbb{Z}, X):=\left\{f \in l^{\infty}(\mathbb{Z}, X): f\right.$ is $\omega$-periodic $\}$, where $\omega \in \mathbb{Z}_{+} \backslash\{0\}$ is fixed.

(vii) $\mathcal{U C}(\mathbb{Z} \times X, X)$ is the set of all functions $f: \mathbb{Z} \times X \rightarrow X$ satisfying that, for all $\epsilon>0$, there exists $\delta>0$ such that $\|f(k, x)-f(k, y)\| \leq \epsilon$ for all $k \in \mathbb{Z}$ and for all $x, y \in X$ with $\|x-y\|<\delta$.

(viii) $\overline{\mathcal{U C}}(\mathbb{Z} \times X, X)$ is the set of all functions $f: \mathbb{Z} \times X \rightarrow X$ satisfying that, for all $\epsilon>0$, there exists $\delta>0$ such that $\|f(k, x)-f(k, y)\| \leq L_{f}(k) \in$ for all $k \in \mathbb{Z}$ and $x, y \in X$ with $\|x-y\| \leq \delta$, where $L_{f} \in l^{p}(\mathbb{Z})$.

Let $h: \mathbb{Z} \rightarrow \mathbb{R}^{+}$be a sequence such that $h(n) \geq 1$ for all $n \in \mathbb{Z}$, and $h(n) \rightarrow \infty$ as $|n| \rightarrow \infty$. Define

$$
\mathcal{C}_{h}^{0}(\mathbb{Z}, X)=\left\{\xi: \mathbb{Z} \rightarrow X: \lim _{|n| \rightarrow \infty} \frac{\|\xi(n)\|}{h(n)}=0\right\},
$$

endowed with the norm $\|\xi\|_{h}=\sup _{n \in \mathbb{Z}} \frac{\|\xi(n)\|}{h(n)}$.

It is clear that $\mathcal{C}_{h}^{0}(\mathbb{Z}, X)$ is a Banach space isometrically isomorphic with the space $C_{0}(\mathbb{Z}, X)$ consisting of all sequences $\xi: \mathbb{Z} \rightarrow X$ that vanish at $\pm \infty$.

We recall a compactness criterion, the Leray-Schauder alternative theorem, and Matkowski's fixed point theorem which will be useful later to prove the existence and uniqueness of solution to (1.3). 
Lemma 2.1 ([34, Lemma 2.1]) Let $h: \mathbb{Z} \rightarrow \mathbb{R}^{+}$be a function such that $h(n) \geq 1$ for all $n \in \mathbb{Z}$ and $h(n) \rightarrow \infty$ as $|n| \rightarrow \infty$. Let $S$ be a subset of $\mathcal{C}_{h}^{0}(\mathbb{Z}, X)$. Suppose that the following conditions are satisfied:

(a) The set $\mathcal{H}(S)=\left\{\frac{u(n)}{h(n)}: u \in S\right\}$ is relatively compact in $X$ for all $n \in \mathbb{Z}$.

(b) $S$ is weighted equiconvergent at $\pm \infty$, that is, for every $\epsilon>0$, there is $T>0$ such that $\|u(n)\|<\epsilon h(n)$ for each $|n| \geq T$ for all $u \in S$.

Then $S$ is relatively compact in $\mathcal{C}_{h}^{0}(\mathbb{Z}, X)$.

Theorem 2.2 ([28, Matkowski's fixed point theorem]) Let $(X, d)$ be a complete metric space, and let $\mathcal{F}: X \rightarrow X$ be a map such that $d(\mathcal{F} x, \mathcal{F} y) \leq \phi(d(x, y))$ for all $x, y \in X$, where $\phi:[0, \infty) \rightarrow[0, \infty)$ is a nondecreasing function such that $\lim _{n \rightarrow \infty} \phi^{n}(t)=0$ for all $t>0$, where $\phi^{n}$ is the nth iterate of $\phi$. Then $\mathcal{F}$ has a unique fixed point $z \in X$.

Theorem 2.3 ([19, Leray-Schauder alternative theorem $]$ ) Let $\Omega$ be a closed convex subset of the Banach space $X$ such that $0 \in \Omega$. Let $\mathcal{F}: \Omega \rightarrow \Omega$ be a completely continuous map. Then the set $\{x \in \Omega: x=\lambda \mathcal{F}(x), 0<\lambda<1\}$ is unbounded or the map $\mathcal{F}$ has a fixed point in $\Omega$.

Let $U$ be the collection of positive sequences $\rho: \mathbb{Z} \rightarrow(0, \infty)$. For $\rho \in U$ and for $n \in \mathbb{Z}_{+}$, we use the notation

$$
\begin{aligned}
& v(n, \rho)=\sum_{k=-n}^{n} \rho(k), \\
& U_{\infty}:=\left\{\rho \in U: \lim _{|n| \rightarrow \infty} v(n, \rho)=\infty\right\}, \\
& U_{b}=\left\{\rho \in U: 0<\inf _{k \in \mathbb{Z}} \rho(k) \leq \sup _{k \in \mathbb{Z}} \rho(k)<\infty\right\} \subset U_{\infty} .
\end{aligned}
$$

Hence, $v(n, \rho)$ are the symmetric partial sums, $U_{\infty}$ consists of those positive sequences $\rho$ over $\mathbb{Z}$ for which the sequence $(v(n, \rho))_{n \in \mathbb{N}}$ is unbounded, while $U_{b}$ consists of the positive sequences $\rho$ such that, for some fixed $\tau>0, \rho(n) \geq \tau$ for all $n \in \mathbb{Z}$.

Let $\rho_{1}, \rho_{2} \in U_{\infty}$ be given. The sequence $\rho_{1}$ is said to be equivalent to $\rho_{2}$ (i.e., $\rho_{1} \sim \rho_{2}$ ) if $\rho_{1} / \rho_{2} \in U_{b}$. It can be proved that $U_{\infty}=\bigcup_{\rho \in U_{\infty}}\left\{\varrho \in U_{\infty}: \rho \sim \varrho\right\}$. For $\rho \in U_{\infty}$ and $m \in \mathbb{Z}$, we define $\rho_{m}(n)=\rho(m+n)$ for $n \in \mathbb{Z}$ and $U_{T}=\left\{\rho \in U_{\infty}: \rho \sim \rho_{m}\right.$ for each $\left.m \in \mathbb{Z}\right\}$.

A sequence $f: \mathbb{Z} \rightarrow X$ is called almost automorphic if, for every integer sequence $\left\{k_{n}^{\prime}\right\}$, there exists a subsequence $\left\{k_{n}\right\}$ such that

$$
\bar{f}(k):=\lim _{n \rightarrow \infty} f\left(k+k_{n}\right)
$$

is well defined for each $k \in \mathbb{Z}$ and $\lim _{n \rightarrow \infty} \bar{f}\left(k-k_{n}\right)=f(k)$. The set of such sequences is denoted by $A A_{d}(\mathbb{Z}, X)$. It is well known that the set $A A_{d}(\mathbb{Z}, X)$ endowed with the norm $\|f\|_{\infty}:=\sup _{k \in \mathbb{Z}}\|f(k)\|$ is a Banach space (see [6]). A function $f: \mathbb{Z} \times X \rightarrow X$ is called almost automorphic if $f(k, x)$ is almost automorphic in $k \in \mathbb{Z}$ for any $x \in X$. We denote the space of all such functions by $A A_{d}(\mathbb{Z} \times X, X)$.

For $\rho_{1}, \rho_{2} \in U_{\infty}$ [33], we define the space

$$
P A A_{0} S\left(\mathbb{Z}, X, \rho_{1}, \rho_{2}\right):=\left\{f \in l^{\infty}(\mathbb{Z}, X): \lim _{n \rightarrow \infty} \frac{1}{v\left(n, \rho_{1}\right)} \sum_{k=-n}^{n}\|f(k)\| \rho_{2}(k)=0\right\} .
$$


Let $\rho_{1}, \rho_{2} \in U_{\infty}$ be given. A sequence $f: \mathbb{Z} \rightarrow X$ is called discrete weighted pseudo almost automorphic if it can be represented as $f=g+\varphi$, where $g \in A A_{d}(\mathbb{Z}, X)$ and $\varphi \in P A A_{0} S\left(\mathbb{Z}, X, \rho_{1}, \rho_{2}\right)$. The space of such functions is denoted by $W P A A_{d}(\mathbb{Z}, X)$. The space $W P A A_{d}(\mathbb{Z}, X)$ endowed with the norm $\|f\|_{\infty}:=\sup _{k \in \mathbb{Z}}\|f(k)\|$ is a Banach space (see [33, Lemma 10]). A function $f: \mathbb{Z} \times X \rightarrow X$ is called discrete weighted almost automorphic in $k \in \mathbb{Z}$ for each $x \in X$ if it can be expressed as $f=g+\varphi$, where $g \in A A_{d}(\mathbb{Z} \times X, X)$ and $\varphi \in P A A_{0} S\left(\mathbb{Z} \times X, X, \rho_{1}, \rho_{2}\right)$. The space of such functions is denoted by $W P A A_{d}(\mathbb{Z} \times X, X)$. In what follows, we denote by $V_{\infty}$ the set of all functions $\rho_{1}, \rho_{2} \in U_{\infty}$ satisfying the following: there exists an unbounded set $\Omega \subset \mathbb{Z}$ such that, for all $m \in \mathbb{Z}$,

$$
\lim _{|k| \rightarrow \infty, k \in \Omega} \sup \frac{\rho_{2}(k+m)}{\rho_{1}(k)}<\infty \quad \text { and } \quad \lim _{n \rightarrow \infty} \frac{\sum_{k \in([-n, n] \backslash \Omega)+m} \rho_{2}(k)}{v\left(n, \rho_{1}\right)}=0 .
$$

A function $f: \mathbb{Z} \times X \rightarrow X$ is said to be locally Lipschitz-continuous with respect to the second variable if, for each positive number $r$, for all $k \in \mathbb{Z}$, and for all $x, y \in X$ with $\|x\| \leq r$ and $\|y\| \leq r$, we have $\|f(k, x)-f(k, y)\| \leq L(r)\|x-y\|$, where $L: \mathbb{R}_{+} \rightarrow \mathbb{R}_{+}$is a nondecreasing function.

A sequence $f \in l^{\infty}(\mathbb{Z}, X)$ is called discrete asymptotically $\omega$-periodic if there exist $g \in$ $C_{\omega}(\mathbb{Z}, X), \varphi \in C_{0}(\mathbb{Z}, X)$ such that $f=g+\varphi$. The collection of all such sequences is denoted by $A P_{\omega}(\mathbb{Z}, X)$. A sequence $f \in l^{\infty}(\mathbb{Z}, X)$ is called discrete S-asymptotically $\omega$-periodic if there exists $\omega \in \mathbb{Z}^{+} \backslash\{0\}$ such that $\lim _{n \rightarrow \infty}(f(n+\omega)-f(n))=0$. The collection of all such sequences is denoted by $S A P_{\omega}(\mathbb{Z}, X)$ (see [33, Definition 5]).

Let $\rho \in U_{\infty}$ be given. A sequence $f \in l^{\infty}(\mathbb{Z}, X)$ is called discrete S-asymptotically $\omega$ periodic if there exists $\omega \in \mathbb{Z}^{+} \backslash\{0\}$ such that $\lim _{n \rightarrow \infty} \frac{1}{2 n} \sum_{k=-n}^{n}\|f(k+\omega)-f(k)\|=0$. The collection of such sequences is denoted by $P S A P_{\omega}(\mathbb{Z}, X)$ (see [33, Definition 6]).

Let $\rho_{1}, \rho_{2} \in U_{\infty}$. A sequence $f \in l^{\infty}(\mathbb{Z}, X)$ is called discrete weighted pseudo Sasymptotically $\omega$-periodic if there exists $\omega \in \mathbb{Z}^{+} \backslash\{0\}$ such that

$$
\lim _{n \rightarrow \infty} \frac{1}{v\left(n, \rho_{1}\right)} \sum_{k=-n}^{n} \rho_{2}(k)\|f(k+\omega)-f(k)\|=0
$$

Denote by $\operatorname{WPSAP}_{\omega}(\mathbb{Z}, X)$ the set of such sequences (see [34, Definition 2.5]). Next, we will recall some properties of $\operatorname{WPSAP}_{\omega}\left(\mathbb{Z}, X, \rho_{1}, \rho_{2}\right)$ proved in [34].

Lemma 2.4 ([34, Lemma 2.2]) Let $\rho_{1}, \rho_{2} \in V_{\infty}$ be given, then

(a) For each $l \in \mathbb{Z}$, one has

$$
\limsup _{n \rightarrow \infty} \frac{v\left(n+l, \rho_{2}\right)}{v\left(n, \rho_{1}\right)}<\infty
$$

(b) $\operatorname{WPSAP}_{\omega}\left(\mathbb{Z}, X, \rho_{1}, \rho_{2}\right)$, where $\omega \in \mathbb{Z}_{+} \backslash\{0\}$, is translation invariant, that is, $f(\cdot+l) \in \operatorname{WPSAP}_{\omega}\left(\mathbb{Z}, X, \rho_{1}, \rho_{2}\right)$ for each $l \in \mathbb{Z}$, iff $\in \operatorname{WPSAP}_{\omega}\left(\mathbb{Z}, X, \rho_{1}, \rho_{2}\right)$.

(c) $\operatorname{WPSAP}_{\omega}\left(\mathbb{Z}, X, \rho_{1}, \rho_{2}\right)$, where $\omega \in \mathbb{Z}_{+} \backslash\{0\}$, is a closed subspace of $l^{\infty}(\mathbb{Z}, X)$.

Remark 2.5 It is easy to see that the following inclusions hold: For $\omega \in \mathbb{Z}_{+} \backslash\{0\}$,

$$
C_{\omega}(\mathbb{Z}, X) \subset A P_{\omega}(\mathbb{Z}, X) \subset S A P_{\omega}(\mathbb{Z}, X) \subset P S A P_{\omega}(\mathbb{Z}, X) \subset \operatorname{WPSAP}_{\omega}(\mathbb{Z}, X) \subset l^{\infty}(\mathbb{Z}, X)
$$


Let $\rho_{1}, \rho_{2} \in U_{\infty}$ be given, and $\omega \in \mathbb{Z}_{+} \backslash\{0\}$. In what follows, we will consider the sets $\mathcal{M}(\mathbb{Z}, X):=\left\{W P A A_{d}(\mathbb{Z}, X), W P S A P_{\omega}(\mathbb{Z}, X)\right\}$ and $\mathcal{M}(\mathbb{Z} \times X, X):=\left\{W P A A_{d}(\mathbb{Z} \times X, X)\right.$, $\left.W_{P S A P}(\mathbb{Z} \times X, X)\right\}$.

\section{Solutions for abstract difference equations of convolution type}

We consider the following abstract difference equation of convolution type:

$$
u(n+1)=\sum_{k=-\infty}^{n} a(n-k) A u(k+1)+\sum_{k=-\infty}^{n} b(n-k) f(k, u(k)), \quad n \in \mathbb{Z},
$$

where $A: D(A) \subset X \rightarrow X$ is a closed linear operator on $X, f \in l^{1}(\mathbb{Z} \times X, X)$, and $a, b: \mathbb{Z}_{+} \rightarrow$ $\mathbb{R}_{+}$are given bounded positive sequences. Note that the associated nonhomogeneous linear equation is given by

$$
u(n+1)=\sum_{k=-\infty}^{n} a(n-k) A u(k+1)+\sum_{k=-\infty}^{n} b(n-k) f(k), \quad n \in \mathbb{Z} .
$$

We begin with the definition of solution for the equations under consideration. We write $[D(A)]$ for the space $D(A)$ equipped with the graph norm (which turns it into a Banach space).

Definition 3.1 Given $a, b \in s(\mathbb{Z}, X)$ bounded positive sequences and $f \in l^{1}(\mathbb{Z}, X)$, a sequence $u: \mathbb{Z} \rightarrow[D(A)]$ is called a strong solution for equation (3.1) if $u \in l^{1}(\mathbb{Z} ;[D(A)])$ and satisfies (3.1).

We now define the concept of discrete resolvent family which will be a crucial tool for the solution of equation (3.1).

Definition 3.2 Let $A$ be a closed linear operator with domain $D(A)$ defined on a Banach space $X$. Let $a$ and $b$ be scalar-valued sequences. An operator-valued sequence $\{S(n)\}_{n \in \mathbb{N}_{0}} \subset \mathcal{B}(X)$ is called a discrete resolvent family generated by $A$ if it satisfies the following conditions:

(i) $S(n)(X) \subset D(A)$, and $S(n) A x=A S(n) x$ for all $x \in D(A)$ and $n \in \mathbb{N}_{0}$;

(ii) $S(n) x=b(n) x+A \sum_{k=0}^{n} a(n-k) S(k) x$ for $n \in \mathbb{N}_{0}$ and $x \in X$.

Remark 3.3 Note that Definition 3.2 corresponds to the resolvent sequence defined in [1, 5] when $b(n)=a(n)=k^{\alpha}(n):=\frac{\Gamma(\alpha+n)}{\Gamma(\alpha) n !}$ for $\alpha>0, n \in \mathbb{N}_{0}$. Sequences of operators for abstract difference equations with the kernel $k^{\alpha}(n)$ were introduced by Lizama in [26] and [25] in connection with abstract difference equations of fractional order.

Remark 3.4 If $a(0)=b(0)=1$, then by Definition 3.2 we have that

$$
S(0) x=x+S(0) A x, \quad x \in D(A)
$$

and

$$
S(0) x=x+A S(0) x, \quad x \in X .
$$


Therefore $1 \in \rho(A)$ and

$$
S(0) x=(I-A)^{-1} x .
$$

Here, $\rho(A)$ denotes the resolvent set of $A$.

Proposition 3.5 If $1 / a(0) \in \rho(A)$, and there exists a discrete resolvent family corresponding to the kernels $a$ and $b$, then it is unique.

Proof Suppose that $S(n)$ and $R(n)$ are resolvent families generated by $A$. Let $x \in X$ and define $\varphi(n)=S(n) x-R(n) x, n \in \mathbb{N}_{0}$. Note that $\varphi(n) \in D(A)$ for all $k \in \mathbb{N}_{0}$ and

$$
\varphi(n)=A \sum_{k=0}^{n} a(n-k) \varphi(k)
$$

Let us consider two cases according to whether $a(0)=0$ or $a(0) \neq 0$. In the case $a(0)=0$, expanding the sum in (3.2), we obtain $\varphi(n)=0$ for all $n \in \mathbb{N}_{0}$. If $a(0) \neq 0$, then by Definition 3.2 and since $1 / a(0) \in \rho(A)$, we obtain $S(0)=b(0)(I-a(0) A)^{-1}=R(0)$. Therefore $\varphi(0)=0$. Using (3.2), we obtain $(I-a(0) A) \varphi(n)=0$ for all $n \in \mathbb{N}_{0}$. Then the invertibility of $(I-a(0) A)$ implies $S(n) x=R(n) x$ for all $n \in \mathbb{N}_{0}$ and $x \in X$.

Remark 3.6 Note that if $b(0) \neq 0$, then $\frac{S(0)}{b(0)}(1-A a(0)) x=x$ for all $x \in D(A)$. Thus combining with Definition 3.2 part (i), we have $1 / a(0) \in \rho(A)$. Then the conclusion of the previous theorem holds.

In what follows $\widehat{a}$ denotes the Laplace transform of the kernel $a$, whenever it exists. We recall [31, Sect. 3.2 p. 69] that a function $a \in L_{\text {loc }}^{1}\left(\mathbb{R}_{+}\right)$of subexponential growth is called $k$-regular if there is a constant $c>0$ such that

$$
\left|\lambda^{n} \widehat{a}^{(n)}(\lambda)\right| \leq c|\widehat{a}(\lambda)| \quad \text { for all } \operatorname{Re} \lambda>0,0 \leq n \leq k
$$

Also $a$ is said to be of positive type if $|\arg \widehat{a}(\lambda)| \leq \frac{\pi}{2}$ for all $\operatorname{Re} \lambda>0$.

Definition 3.7 Let $a \in L_{\text {loc }}^{1}\left(\mathbb{R}_{+}\right)$and $k \geq 2$. We say that $a(t)$ is $k$-monotone if $a \in$ $C^{k-2}(0, \infty),(-1)^{n} a^{(n)}(t) \geq 0$ for all $t>0,0 \leq n \leq k-2$, and the function $(-1)^{k-2} a^{(k-2)}(t)$ is nonincreasing and convex.

Theorem 3.8 Suppose that A generates a discrete resolvent family $\{S(n)\}_{n \in \mathbb{N}_{0}} \subset \mathcal{B}(X)$ with $a(n)=b(n) \in s\left(\mathbb{N}_{0}, \mathbb{R}^{+}\right)$such that $1 / a(0) \in \rho(A)$. Then the following statements hold:

(a) For all $x \in X$, we have that $S(0) x=a(0) R(A) x, S(1) x=a(1) R^{2}(A) x$, and

$$
S(n) x=\left[\sum_{j=0}^{n-1} \phi_{j}(n)(A R(A))^{j}\right] R^{2}(A) x, \quad n \geq 2,
$$

where $R(A):=(1-a(0) A)^{-1}$ and $\phi_{0}(n)=a(n), \phi_{1}(n)=\sum_{k=1}^{n-1} a(n-k) a(k)$, and

$$
\phi_{j}(n)=\sum_{k=j}^{n-1} a(n-k) \phi_{j-1}(k), \quad j \geq 2 .
$$


(b) $S(n) X \subset D(A)$ and $S(n) A x=A S(n) x$ for all $x \in D(A)$, and $n \in \mathbb{N}_{0}$.

Proof Clearly, by Definition 3.2 part (ii), we obtain that

$$
S(0) x=a(0) R(A) x .
$$

Now, let $x \in X$, we have that

$$
S(1) x=a(1) x+A(a(1) S(0)+a(0) S(1)),
$$

then

$$
S(1)(1-a(0) A) x=a(1) x+a(1) a(0) A R(A) x=a(1) R(A) x .
$$

Thus

$$
S(1) x=a(1) R^{2}(A) x,
$$

where we have used that $A R(A)=\frac{1}{a(0)}(-1+R(A))$. By induction, we suppose that

$$
S(m) x=\left[\sum_{j=0}^{m-1} \phi_{j}(m)(A R(A))^{j}\right] R^{2}(A) x \quad \text { for } m \leq n-1 .
$$

For $x \in X$, we write, using the definition:

$$
\begin{aligned}
S(n) x & =a(n) x+A \sum_{m=0}^{n} a(n-m) S(m) x \\
& =a(n) x+A[a(n) S(0)+a(n-1) S(1)+a(0) S(n)] x+A \sum_{m=2}^{n-1} a(n-m) S(m) x .
\end{aligned}
$$

Then we obtain

$$
(1-a(0) A) S(n)=a(n)(1+A S(0))+A a(n-1) a(1) R^{2}(A)+A \sum_{m=2}^{n-1} a(n-m) S(m) .
$$

Therefore, since $R(A)(1+A S(0))=R^{2}(A)$, we obtain

$$
\begin{aligned}
S(n) x= & a(n) R^{2}(A) x+A a(n-1) a(1) R^{3}(A) x+A \sum_{m=2}^{n-1} a(n-m) S(m) R(A) x \\
= & a(n) R^{2}(A) x+a(n-1) a(1) A R^{3}(A) x \\
& +\sum_{m=2}^{n-1} a(n-m)\left[a(m) A R^{3}(A) x+\sum_{j=1}^{m-1} \phi_{j}(m) A^{j+1} R^{j+3}(A)\right] x \\
= & a(n) R^{2}(A) x+a(n-1) a(1) A R^{3}(A) x+A R^{3}(A) \sum_{m=2}^{n-1} a(n-m) a(m) x
\end{aligned}
$$




$$
\begin{aligned}
& +\sum_{m=2}^{n-1} a(n-m) \sum_{j=1}^{m-1} \phi_{j}(m) A^{j+1} R^{j+3}(A) x \\
= & a(n) R^{2}(A) x+\sum_{m=1}^{n-1} a(n-m) a(m) A R^{3}(A) x+\sum_{m=2}^{n-1} a(n-m) \sum_{j=1}^{m-1} \phi_{j}(m) A^{j+1} R^{j+3}(A) x \\
= & a(n) R^{2}(A) x+\phi_{1}(n) A R^{3}(A) x+\sum_{m=2}^{n-1} a(n-m) \sum_{j=1}^{m-1} \phi_{j}(m) A^{j+1} R^{j+3}(A) x \\
= & {\left[\sum_{j=0}^{n-1} \phi_{j}(n)(A R(A))^{j}\right] R^{2}(A) x . }
\end{aligned}
$$

Next, we prove (b). Note that

$$
A R^{j}(A)=\left(A-\frac{1}{a(0)}+\frac{1}{a(0)}\right) R^{j}(A)=\frac{1}{a(0)}\left(R^{j}(A)-R^{j-1}(A)\right)
$$

and

$$
R^{j}(A) A=R^{j}(A)\left(A-\frac{1}{a(0)}+\frac{1}{a(0)}\right)=\frac{1}{a(0)}\left(R^{j}(A)-R^{j-1}(A)\right) .
$$

Therefore, for all $x \in X$, it is a straightforward consequence of the above representation of $S(n)$ that (b) holds.

The next theorem gives necessary conditions in terms of $C_{0}$-semigroups in order to ensure the existence and summability of a discrete resolvent family. We will denote by $f * g:=\int_{0}^{t} f(t-s) g(s) d s$ the Laplace convolution of the functions $f$ and $g$, and $\rho_{n}(t)$ will be the function $\rho_{n}(t)=\frac{e^{-t} t^{n}}{n !}$. The notion of 2-regular function is taken from the reference [31].

Theorem 3.9 Let $A$ be the generator of a bounded analytic $C_{0}$-semigroup on a Banach space X. Let $k(t), g(t) \geq 0$ be given by $a(n)=\int_{0}^{\infty} \rho_{n}(t) k(t) d t, b(n)=\int_{0}^{\infty} \rho_{n}(t) g(t) d t$, where $k \in L_{\text {loc }}^{1}\left(\mathbb{R}_{+}\right)$is 2-regular and of subexponential growth, of positive type, such that $\frac{1}{\lambda k(\lambda)}$ defined for $\lambda \neq 0$ has a locally analytic extension at $\lambda=0, g(0)=0, g \in W^{1,1}\left(\mathbb{R}_{+}\right)$, and $0 \in$ $\rho(A)$. Then A generates a summable discrete resolvent family $\{R(n)\}_{n \in \mathbb{N}_{0}}$, with sequences $b(n)$ and $a(n)$.

Proof By [31, Corollary 3.1] $\widehat{k}(\lambda) \neq 0, \frac{1}{\hat{k}(\lambda)} \in \rho(A)$ for all $\operatorname{Re} \lambda>0$, and there exists a constant $M \geq 1$ such that $H(\lambda)=(I-\widehat{k}(\lambda) A)^{-1} / \lambda$ satisfies $\|H(\lambda)\| \leq \frac{M}{|\lambda|}$ for all $\operatorname{Re} \lambda>0$. Then, by [31, Theorem 10.2], there exists a resolvent family $S(t)$ which is uniformly integrable. By [31, Definition 1.3, (S3)] for all $x \in X$ and $t>0$, we have that

$$
S(t) x=x+A(k * S)(t) x .
$$

Define $T(t) x:=\left(g^{\prime} * S\right)(t) x$ for all $x \in X$ and $t \geq 0$. Note that $T(t) \in L^{1}\left(\mathbb{R}_{+}, X\right)$ since $S(t)$ is uniformly integrable and $g^{\prime} \in L^{1}\left(\mathbb{R}_{+}\right)$. Moreover, since $g(0)=0$, it follows that $\left(g^{\prime} * 1\right)(t)=$ $\int_{0}^{t} g^{\prime}(s) d s=g(t)$. Thus, for all $x \in X$ and $t \geq 0$, we obtain from (3.3)

$$
T(t) x=\left(g^{\prime} * 1\right)(t) x+A\left(k * g^{\prime} * S\right)(t) x
$$




$$
=g(t) x+A(k * T)(t) x .
$$

Define

$$
R(n) x:=\int_{0}^{\infty} \rho_{n}(t) T(t) x d t=\left.\frac{(-1)^{n}}{n !}\left[\frac{\widehat{g}(\lambda)}{\widehat{k}(\lambda)}\left(\frac{1}{\widehat{k}(\lambda)}-A\right)^{-1} x\right]^{(n)}\right|_{\lambda=1} \quad \text { for all } n \in \mathbb{N}_{0}, x \in X .
$$

Then $R(n) x \in D(A)$ for all $x \in X$. Now, from $a(n)=\int_{0}^{\infty} \rho_{n}(t) a(t) d t$ and $b(n)=$ $\int_{0}^{\infty} \rho_{n}(t) g(t) d t$, and using [26, Theorem 3.4], we have that, for all $x \in X$,

$$
R(n) x=b(n) x+A \sum_{j=0}^{n} a(n-j) R(j) x .
$$

Finally, we prove that $R(n)$ is summable. In fact, since $T(t) \in L^{1}\left(\mathbb{R}_{+}, X\right)$, we have that

$$
\begin{aligned}
\sum_{n=0}^{\infty}\|R(n)\| & =\sum_{n=0}^{\infty}\left\|\int_{0}^{\infty} \rho_{n}(t) T(t) d t\right\|=\sum_{n=0}^{\infty}\left\|\int_{0}^{\infty} \frac{e^{-t} t^{n}}{n !} T(t) d t\right\| \\
& \leq \int_{0}^{\infty}\|T(t)\| d t<\infty .
\end{aligned}
$$

Remark 3.10 We note that the family $T$ defined in the previous theorem is the convolution of a function $g^{\prime}$ with a resolvent family $S$. The resolvent families have been studied extensively by Prüss in [31]. It is well known that under certain conditions on the function $k$, we can obtain resolvent families with various additional properties: analytic, differentiable, exponentially bounded, uniform integrable, among others, see [31]. Next, we will give conditions on $g$ in order that the family $T$ have the same properties of $S$.

- By Young's inequality, if $S(t)$ is uniformly integrable and $g^{\prime} \in L^{1}\left(\mathbb{R}_{+}\right)$, then $T(t) \in L^{1}\left(\mathbb{R}_{+}, X\right)$.

- Suppose that $S$ is differentiable, then by [31, Definition 1.4] we see that $S(\cdot) x \in W_{\text {loc }}^{1,1}\left(\mathbb{R}_{+}, X\right)$ for each $x \in D(A)$, and there is $\varphi \in L_{\text {loc }}^{1}\left(\mathbb{R}_{+}\right)$such that $\left\|S^{\prime}(t) x\right\| \leq \varphi(t)\|x\|_{A}$ a.e. on $\mathbb{R}_{+}$, for each $x \in D(A)$. If $g \in W_{\text {loc }}^{1,1}\left(\mathbb{R}_{+}\right)$, then $T(t) \in W_{\text {loc }}^{1,1}\left(\mathbb{R}_{+}\right)$. Moreover,

$$
\begin{aligned}
\left\|\frac{d}{d t}\left(g^{\prime} * S\right)(t) x\right\| & \leq\left\|S(0) g^{\prime}(t) x\right\|+\left\|\int_{0}^{t} S^{\prime}(t-s) g^{\prime}(s) x d s\right\| \\
& \leq M|x|_{A}+M \int_{0}^{t}\left\|S^{\prime}(t-s) x\right\| d s \\
& \leq M|x|_{A}+M \int_{0}^{t} \varphi(t-s)\|x\|_{A} d s \leq(M+K)\|x\|_{A},
\end{aligned}
$$

where $M=\sup _{t \in \mathbb{R}_{+}}\left|g^{\prime}(t)\right|$ and $K=M \int_{0}^{t} \varphi(t-s) d s$. Thus $T(t)$ is differentiable.

Remark 3.11 The conditions imposed on the sequences $a(n)$ and $b(n)$ in Theorem 3.9 are sufficient but not necessary. In [1, Theorem 3.5] the authors proved that if $0<\alpha<1$ and $A$ is the generator of an exponentially stable $C_{0}$-semigroup $\{T(t)\}_{t \geq 0}$, defined on a Banach space $X$, then $A$ generates a summable discrete resolvent family $\left\{S_{\alpha}(n)\right\}_{n \in \mathbb{N}_{0}}$ defined by

$$
S_{\alpha}(n) x:=\int_{0}^{\infty} \int_{0}^{\infty} e^{-t} \frac{t^{n}}{n !} f_{s, \alpha}(t) T(s) x d s d t, \quad n \in \mathbb{N}_{0}, x \in X,
$$


where $f_{t, \alpha}(\lambda)$ is a probability density function frequently called stable Lévy process. The latter is defined by [35]

$$
f_{t, \alpha}(\lambda)=\frac{1}{2 \pi i} \int_{\sigma-i \infty}^{\sigma+i \infty} e^{z \lambda-t z^{\alpha}} d z, \quad \sigma>0, t>0, \lambda>0,0<\alpha<1,
$$

where the branch of $z^{\alpha}$ is so taken that $\operatorname{Re}\left(z^{\alpha}\right)>0$ for $\operatorname{Re}(z)>0$.

Let $f, g$ be sequences (defined on $\mathbb{Z}_{+}$). We define two convolution products $(f \circ g)(n)$ and $(f * g)(n)$ as follows:

$$
(f \circ g)(n)=\sum_{k=-\infty}^{n} f(n-k) g(k) \quad \text { and } \quad(f * g)(n)=\sum_{k=0}^{n} f(n-k) g(k) \text {. }
$$

For the second product, no condition on the sequences is required, while for the first we may for example assume that one of the sequences is summable and the other is bounded. In the sequel, we derive a result which describes properties of the above operations.

Theorem 3.12 For $f, g$, $h$ given sequences, the following properties hold:

(i) $(f * g)(n)=(g * f)(n)$.

(ii) $((f * g) \circ h)(n)=(g \circ(f \circ h))(n)$.

(iii) $(g \circ(f \circ h))(n)=(f \circ(g \circ h))(n)$.

Proof (i) is obvious. Now, we will prove (ii). By Fubini's theorem,

$$
\begin{aligned}
((f * g) \circ h)(n) & =\sum_{j=-\infty}^{n}(f * g)(n-j) h(j)=\sum_{j=-\infty}^{n} \sum_{k=0}^{n-j} f(n-j-k) g(k) h(j) \\
& =\sum_{k=0}^{\infty} g(k) \sum_{j=-\infty}^{n-k} f(n-j-k) h(j)=\sum_{k=0}^{\infty} g(k)(f \circ h)(n-k) \\
& =\sum_{j=-\infty}^{n}(f \circ h)(j) g(n-j)=(g \circ(f \circ h))(n) .
\end{aligned}
$$

This proves (ii). Now, (iii) follows from (i) and (ii).

The following theorem gives conditions for better regularity.

Theorem 3.13 Let $\{S(n)\}_{n \in \mathbb{N}_{0}} \subset \mathcal{B}(X)$ be a summable discrete resolvent family generated by $A$ and $f \in l^{1}(\mathbb{Z},[D(A)])$, then

$$
u(n+1):=\sum_{k=-\infty}^{n} S(n-k) f(k)
$$

is a strong solution of (3.1). 
Proof Note that $u$ is clearly well defined and $u \in l^{1}(\mathbb{Z},[D(A)])$. Now we will prove that $u$ satisfies (3.1). Indeed, by Definition 3.2 and Theorem 3.12,

$$
\begin{aligned}
& u(n+1)-\sum_{k=-\infty}^{n} b(n-k) f(k) \\
& =(S \circ f)(n)-(b \circ f)(n)=\sum_{k=-\infty}^{n} S(n-k) f(k)-(b \circ f)(n) \\
& =\sum_{k=-\infty}^{n}\left[b(n-k)+A \sum_{l=0}^{n-k} a(n-k-l) S(l)\right] f(k)-(b \circ f)(n) \\
& =(b \circ f)(n)+A(a * S \circ f)(n)-(b \circ f)(n) \\
& =A \sum_{k=-\infty}^{n} a(n-j) u(j+1) .
\end{aligned}
$$

Thus,

$$
u(n+1)=\sum_{k=-\infty}^{n} b(n-j) f(j)+A \sum_{k=-\infty}^{n} a(n-j) u(j+1) .
$$

This proves the claim.

Example 3.14 Consider the special case $a(n)=b(n)=k^{\alpha}(n), n \in \mathbb{Z}$, and $\{S(n)\}_{n \in \mathbb{N}}$ satisfying Definition 3.2, then the nonlinear fractional difference equation

$$
\Delta^{\alpha} u(n)=A u(n+1)+f(n), \quad n \in \mathbb{Z},
$$

for $0<\alpha<1$, can be written in the form (3.1). Here, $A$ is the generator of an $\alpha$ resolvent sequence $\{S(n)\}_{n \in \mathbb{N}_{0}}$ in $\mathcal{B}(X), \Delta^{\alpha}$ denotes fractional difference in Weyl-like sense (see $[1$, 5]), and $f$ satisfies Lipschitz conditions of global and local type.

Indeed, first we recall the following definitions.

Definition 3.15 ([1]) Let $\alpha>0$ be given and $\rho(n)=|n|^{\alpha-1}, n \in \mathbb{Z}$. The $\alpha$ th fractional sum of a sequence $f \in l_{\rho}^{1}(\mathbb{Z}, X)$ is defined by

$$
\Delta^{-\alpha} f(n):=\sum_{j=-\infty}^{n} k^{\alpha}(n-j) f(j), \quad n \in \mathbb{Z} .
$$

Definition 3.16 ([1]) Let $\alpha>0$ be given and $\rho(n)=|n|^{\alpha-1}, n \in \mathbb{Z}$. The $\alpha$ th fractional difference of a sequence $f \in l_{\rho}^{1}(\mathbb{Z}, X)$ is defined by

$$
\Delta^{\alpha} f(n):=\Delta^{m} \Delta^{-(m-\alpha)} f(n), \quad n \in \mathbb{Z},
$$

where $m=[\alpha]+1,[\cdot]$ is the greatest integer function. 
Now if

$$
\Delta^{\alpha} u(n)=A u(n+1)+f(n),
$$

then

$$
\sum_{k=-\infty}^{n} k^{\alpha}(n-k) \Delta^{\alpha} u(k)=A \sum_{k=-\infty}^{n} k^{\alpha}(n-k) u(k+1)+\sum_{k=-\infty}^{n} k^{\alpha}(n-k) f(k) .
$$

Note that

$$
\begin{aligned}
& \sum_{k=-\infty}^{n} k^{\alpha}(n-k)\left(k^{1-\alpha} * u\right)(k+1) \\
& \quad=\sum_{m=-\infty}^{n+1} k^{\alpha}(n-m+1)\left(k^{1-\alpha} \circ u\right)(m)=\left(k^{\alpha} \circ\left(k^{1-\alpha} \circ u\right)\right)(m+1) \\
& \quad=\left(\left(k^{\alpha} * k^{1-\alpha}\right) \circ u\right)(m+1)=\sum_{k=-\infty}^{m+1} u(k) .
\end{aligned}
$$

Analogously,

$$
\sum_{k=-\infty}^{n} k^{\alpha}(n-k)\left(k^{1-\alpha} * u\right)(k)=\sum_{k=-\infty}^{m} u(k)
$$

Thus,

$$
\begin{aligned}
\sum_{k=-\infty}^{n} k^{\alpha}(n-k) \Delta^{\alpha} u(k) & =\sum_{k=-\infty}^{n} k^{\alpha}(n-k)\left[\left(k^{1-\alpha} * u\right)(k+1)-\left(k^{1-\alpha} * u\right)(k)\right] \\
& =\sum_{k=-\infty}^{n+1} u(k)-\sum_{k=-\infty}^{n} u(k)=u(n+1) .
\end{aligned}
$$

Then

$$
u(n+1)=A \sum_{k=-\infty}^{n} k^{\alpha}(n-k) u(k+1)+\sum_{k=-\infty}^{n} k^{\alpha}(n-k) f(k) .
$$

More information on the sequences $k^{\alpha}(n)$ can be found in the reference [26], in particular the semi group identity $k^{\alpha} * k^{\beta}=k^{\alpha+\beta}, \alpha, \beta>0$.

In the next result, borrowing ideas from [5], we prove regularity under convolution in the above mentioned spaces.

Theorem 3.17 Let $\rho_{1}, \rho_{2} \in V_{\infty}$ and $\rho \in U_{T}$ be given. Assume that A generates a summable discrete resolvent family $\{S(n)\}_{n \in \mathbb{N}_{0}} \subset \mathcal{B}(X)$. Iff belongs to one of the spaces $\Omega \in \mathcal{M}(\mathbb{Z}, X)$, then the sequence $u$ defined by

$$
u(n+1)=\sum_{k=-\infty}^{n} S(n-k) f(k), \quad n \in \mathbb{Z}
$$

belongs to the same space $\Omega$. 
Proof Note that $u(n+1)$ is well defined and $\|u(n+1)\| \leq\|S\|_{1}\|f\|_{\infty}$ for all $n \in \mathbb{Z}$. First, we consider $f \in W P A A_{d}(\mathbb{Z}, X)$. Let $f=f_{1}+f_{2}$, where $f_{1} \in A A_{d}(\mathbb{Z}, X)$ and $f_{2} \in$ $P A A_{0} S\left(\mathbb{Z}, X, \rho_{1}, \rho_{2}\right)$ is the decomposition of $f$. Then

$$
u(n)=\sum_{k=-\infty}^{n-1} S(n-1-k) f_{1}(k)+\sum_{k=-\infty}^{n-1} S(n-1-k) f_{2}(k)=: u_{1}(n)+u_{2}(n) .
$$

From [6, Theorem 2.12], $u_{1} \in A A_{d}(\mathbb{Z}, X)$. Now, we will prove that $u_{2} \in P A A_{0} S\left(\mathbb{Z}, X, \rho_{1}, \rho_{2}\right)$. Indeed,

$$
\begin{aligned}
& \frac{1}{v\left(K, \rho_{1}\right)} \sum_{k=-K}^{K}\left\|u_{2}(k)\right\| \rho_{2}(k) \\
& =\frac{1}{v\left(K, \rho_{1}\right)} \sum_{k=-K}^{K}\left\|\sum_{j=-\infty}^{k-1} S(k-1-j) f_{2}(j)\right\| \rho_{2}(k) \\
& \leq \sum_{m=0}^{\infty}\|S(m)\|\left(\frac{1}{v\left(K, \rho_{1}\right)} \sum_{k=-K}^{K}\left\|f_{2}(k-1-m)\right\| \rho_{2}(k)\right) .
\end{aligned}
$$

By [33, Lemma 10] the space $P A A_{0} S\left(\mathbb{Z}, X, \rho_{1}, \rho_{2}\right)$ is invariant under translations, then $f_{2}(\cdot-m) \in P A A_{0} S\left(\mathbb{Z}, X, \rho_{1}, \rho_{2}\right)$. Thus, by the Lebesgue dominated convergence theorem, we obtain

$$
\lim _{K \rightarrow \infty} \frac{1}{v\left(K, \rho_{1}\right)} \sum_{k=-K}^{K}\left\|u_{2}(k)\right\| \rho_{2}(k)=0
$$

This proves the claim for such a space. Now, let $f \in \operatorname{WPSAP}_{\omega}\left(\mathbb{Z}, X, \rho_{1}, \rho_{2}\right)$. Then there exists $\omega \in \mathbb{Z}^{+} \backslash\{0\}$ such that

$$
\lim _{n \rightarrow \infty} \frac{1}{v\left(n, \rho_{1}\right)} \sum_{k=-n}^{n} \rho_{2}(k)\|f(k+\omega)-f(k)\|=0
$$

Now, we have

$$
\begin{aligned}
& \frac{1}{v\left(m, \rho_{1}\right)} \sum_{n=-m}^{m}\|u(n+\omega)-u(n)\| \rho_{2}(n) \\
& \leq \frac{1}{v\left(m, \rho_{1}\right)} \sum_{n=-m}^{m} \sum_{k=-\infty}^{n-1}\|S(n-1-k)\|\|f(k+\omega)-f(k)\| \rho_{2}(n) \\
& \leq \sum_{k=0}^{\infty}\|S(k)\|\left(\frac{1}{v\left(m, \rho_{1}\right)} \sum_{n=-m}^{m}\|f(n-1-k+\omega)-f(n-1-k)\| \rho_{2}(n)\right) .
\end{aligned}
$$

By [34, Lemma 2.2] $\operatorname{WPSAP}_{\omega}(\mathbb{Z}, X)$ is invariant under translations. Thus, applying again the Lebesgue dominated convergence theorem, we obtain

$$
\lim _{m \rightarrow \infty} \frac{1}{v\left(m, \rho_{1}\right)} \sum_{n=-m}^{m}\|u(n+\omega)-u(n)\| \rho_{2}(n)
$$




$$
\leq \lim _{m \rightarrow \infty} \sum_{k=0}^{\infty}\|S(k)\|\left(\frac{1}{v\left(m, \rho_{1}\right)} \sum_{n=-m}^{m}\|f(n-1-k+\omega)-f(n-1-k)\| \rho_{2}(n)\right)=0 .
$$

\section{Semilinear difference equations}

In this section we use the above defined resolvent families to investigate the existence and uniqueness of solutions for the following class of abstract semilinear difference equations:

$$
u(n+1)=A \sum_{k=-\infty}^{n} a(n-k) u(k+1)+\sum_{k=-\infty}^{n} b(n-k) f(k, u(k)), \quad n \in \mathbb{Z}
$$

where $A$ is the generator of a discrete resolvent family $\{S(n)\}_{n \in \mathbb{N}_{0}}$ in $\mathcal{B}(X), f: \mathbb{Z} \times X \rightarrow X$ is a function bounded on bounded subsets of $X$, and $a, b$ are given such that (4.1) makes sense.

We introduce the following conditions in order to prove our main results about the asymptotic behavior of mild solutions.

(H2) $A$ is the generator of a summable discrete resolvent family $\{S(n)\}_{n \in \mathbb{N}_{0}} \subset \mathcal{B}(X)$.

(F1) $f$ satisfies the Lipschitz condition

$$
\|f(k, h(k) u)-f(k, h(k) v)\| \leq L_{f}\|u-v\| \quad \text { for all } k \in \mathbb{Z}, u, v \in X,
$$

where $L_{f}>0$ is a constant and $h$ is given in Lemma 2.1.

(F2) $f$ satisfies the local Lipschitz condition, that is, for each positive number $r$ and all $u, v \in X$ with $\|u\| \leq r,\|v\| \leq r$, we have

$$
\|f(k, h(k) u)-f(k, h(k) v)\| \leq L_{f}(r)\|u-v\| \quad \text { for all } k \in \mathbb{Z},
$$

where $L_{f}: \mathbb{R}^{+} \rightarrow \mathbb{R}^{+}$is a nondecreasing function and $h$ is given in Lemma 2.1.

(F3) $f$ satisfies the following condition:

$$
\|f(k, h(k) u)-f(k, h(k) v)\| \leq L_{f}(k)\|u-v\| \quad \text { for all } k \in \mathbb{Z}, u, v \in X,
$$

where $L_{f}: \mathbb{Z} \rightarrow \mathbb{R}^{+}$is a summable function and $h$ is given in Lemma 2.1.

(F4) $f$ satisfies

$$
\|f(k, h(k) u)-f(k, h(k) v)\| \leq \phi(\|u-v\|) \quad \text { for all } k \in \mathbb{Z}, u, v \in X,
$$

where $\phi: \mathbb{R}^{+} \rightarrow \mathbb{R}^{+}$is a nondecreasing function and $h$ is given in Lemma 2.1.

(F5) $f$ satisfies

$$
\|f(k, u)-f(k, v)\| \leq L_{f}(k)\|u-v\|^{\theta} \quad \text { for all } k \in \mathbb{Z}, u, v \in X,
$$

where $\theta \in(0,1), L_{f}: \mathbb{Z} \rightarrow \mathbb{R}^{+}$is a sequence (depending on $f$ ) such that $\lim _{|k| \rightarrow \infty} L_{f}(k)=0$.

Next we introduce the definition of solution for the semilinear difference equation.

Definition 4.1 Let $A$ be the generator of a discrete resolvent family $\{S(n)\}_{n \in \mathbb{N}_{0}} \subset \mathcal{B}(X)$ and $f: \mathbb{Z} \times X \rightarrow X$. We say that a sequence $u: \mathbb{Z} \rightarrow \mathbb{X}$ is a mild solution of (4.1) if $k \rightarrow$ 
$S(n-k) f(k, u(k))$ is summable on $\mathbb{N}_{0}$ for each $n \in \mathbb{Z}$ and $u$ satisfies

$$
u(n+1)=\sum_{k=-\infty}^{n} S(n-k) f(k, u(k)), \quad n \in \mathbb{Z}
$$

In the next Theorems 4.2, 4.3, and 4.4, we show existence, uniqueness, and asymptotic behavior of discrete mild solutions of (4.1). We assume that $f$ satisfies Lipschitz and locally Lipschitz conditions, the proofs are based on the Banach fixed point theorem.

Theorem 4.2 Assume that (H2), (F1) hold and $L_{f}\|S\|_{1}<1$, then there exists a unique mild solution $u(n)$ of (4.1) such that $\lim _{|n| \rightarrow \infty} \frac{\|u(n)\|}{h(n)}=0$.

Proof Consider the operator $F: \mathcal{C}_{h}^{0}(\mathbb{Z}, X) \rightarrow \mathcal{C}_{h}^{0}(\mathbb{Z}, X)$ defined by

$$
(F u)(n):=\sum_{k=-\infty}^{n-1} S(n-1-k) f(k, u(k)), \quad n \in \mathbb{Z}
$$

Note that $F$ is well defined. Indeed,

$$
\begin{aligned}
\|(F u)(n)\| & \leq \sum_{k=-\infty}^{n-1}\|S(n-1-k)\|\|f(k, u(k))-f(k, 0)\|+\sum_{k=-\infty}^{n-1}\|S(n-1-k)\|\|f(k, 0)\| \\
& \leq L_{f}\|S\|_{1}\|u\|_{h}+\|S\|_{1} \sup _{k \in \mathbb{Z}}\|f(k, 0)\|,
\end{aligned}
$$

hence $\lim _{|n| \rightarrow \infty} \frac{\|(F u)(n)\|}{h(n)}=0$, which implies that $F$ is well defined. In addition, for $u, v \in$ $\mathcal{C}_{h}^{0}(\mathbb{Z}, X)$ and $n \in \mathbb{Z}$, the following inequality holds:

$$
\|(F u)(n)-(F v)(n)\| \leq \sum_{k=-\infty}^{n-1}\|S(n-1-k)\|\|f(k, u(k))-f(k, v(k))\| \leq L_{f}\|S\|_{1}\|u-v\|_{h}
$$

therefore, $\|F u-F v\|_{h} \leq L_{f}\|S\|_{1}\|u-v\|_{h}$. From the assumption $L_{f}\|S\|_{1}<1$ we see that $F$ is a contraction, and using the Banach fixed point theorem, we conclude that there exists a unique discrete mild solution of (4.1) such that $\lim _{|n| \rightarrow \infty} \frac{\|u(n)\|}{h(n)}=0$.

In the following theorem, we make a modification on the previous hypothesis on the Lipschitz condition, namely we consider a local condition instead of the global one.

Theorem 4.3 Suppose (H2), (F2) and that there exists $r_{0}>0$ such that $\|S\|_{1}\left(L_{f}\left(r_{0}\right)+\right.$ $\left.\frac{1}{r_{0}} \sup _{k \in \mathbb{Z}}\|f(k, 0)\|\right)<1$, then there exists a unique mild solution $u(n)$ of (4.1) such that $\lim _{|n| \rightarrow \infty} \frac{\|u(n)\|}{h(n)}=0$.

Proof Define the operator $F$ as in (4.2), then $H$ is well defined. Let

$$
B_{r_{0}}:=\left\{u \in \mathcal{C}_{h}^{0}(\mathbb{Z}, X):\|u\|_{h} \leq r_{0}\right\}
$$

For $u \in B_{r_{0}}$

$$
\|(F u)(n)\| \leq\|S\|_{1}\left(L_{f}\left(r_{0}\right)+\frac{1}{r_{0}} \sup _{k \in \mathbb{Z}}\|f(k, 0)\|\right) r_{0} \leq r_{0} .
$$


Therefore, $\|F u\| \leq r_{0}$, that is, $F u \in B_{r_{0}}$. Moreover, for $u, v \in B_{r_{0}}$, we have

$$
\|(F u)(n)-(F v)(n)\| \leq L_{f}\left(r_{0}\right)\|S\|_{1}\|u-v\|_{h},
$$

then there is a unique fixed point $u \in B_{r_{0}}$, so there is a unique mild solution $u(n)$ of (4.1) such that $\lim _{|n| \rightarrow \infty} \frac{\|u(n)\|}{h(n)}=0$.

Theorem 4.4 Assume that (H2), (F3) hold, then there exists a unique mild solution $u(n)$ of (3.1) such that $\lim _{|n| \rightarrow \infty} \frac{\|u(n)\|}{h(n)}=0$.

Proof Define the operator $F$ as in (4.2). Then by hypothesis (F4), we have that

$$
\begin{aligned}
\|(F u)(n)\| & \leq \sum_{k=-\infty}^{n-1}\|S(n-1-k)\| L_{f}(k) \frac{\|u(k)\|}{h(k)}+\|S\|_{1} \sup _{k \in \mathbb{Z}}\|f(k, 0)\| \\
& \leq\|S\|_{\infty}\left\|L_{f}\right\|_{1}\|u\|_{h}+\|S\|_{1} \sup _{k \in \mathbb{Z}}\|f(k, 0)\|,
\end{aligned}
$$

where $\left\|L_{f}\right\|_{1}:=\sum_{k=-\infty}^{\infty} L_{f}(k)$, so $F$ is well defined.

For $u, v \in \mathcal{C}_{h}^{0}(\mathbb{Z}, X)$, one has

$$
\|(F u)(n)-(F v)(n)\| \leq\|S\|_{\infty}\left(\sum_{k=-\infty}^{n-1} L_{f}(k)\right)\|u-v\|_{h} .
$$

Similarly, by [14, Lemma 3.2],

$$
\begin{aligned}
\left\|\left(F^{2} u\right)(n)-\left(F^{2} v\right)(n)\right\| & \leq \sum_{k=-\infty}^{\infty} L_{f}(k)\|S(n-1-k)\| \frac{\|(F u)(k)-(F v)(k)\|}{h(k)} \\
& \leq\left(\|S\|_{\infty}\right)^{2}\left(\sum_{k=-\infty}^{n-1} L_{f}(k)\left(\sum_{j=-\infty}^{k-1} L_{f}(j)\right)\right)\|u-v\|_{h} \\
& \leq \frac{\left(\|S\|_{\infty}\right)^{2}}{2 !}\left(\sum_{k=-\infty}^{\infty} L_{f}(k)\right)^{2}\|u-v\|_{h} .
\end{aligned}
$$

By induction, one can easily see that

$$
\left\|\left(F^{n} u\right)(n)-\left(F^{n} v\right)(n)\right\| \leq \frac{\left(\|S\|_{\infty}\left\|L_{f}\right\|_{1}\right)^{n}}{n !}\|u-v\|_{h} .
$$

Therefore, $\left\|F^{n} u-F^{n} v\right\|_{h} \leq \frac{\left(\|S\|_{\infty}\left\|L_{f}\right\|_{1}\right)^{n}}{n !}\|u-v\|_{h}$. For sufficiently large $n$, we have $\frac{\left(\|S\|_{\infty}\left\|L_{f}\right\|_{1}\right)^{n}}{n !}<1$. By the Banach contraction mapping principle, $F$ has a unique fixed point in $\mathcal{C}_{h}^{0}(\mathbb{Z}, X)$, so there is a unique mild solution $u(n)$ of (3.1) such that $\lim _{|n| \rightarrow \infty} \frac{\|u(n)\|}{h(n)}=0$.

The following theorem establishes the existence of a mild solution of (4.1) in $\mathcal{C}_{h}^{0}(\mathbb{Z}, X)$, based on the fixed point theorem of Matkowski.

Theorem 4.5 Let (H2), (F4) hold and assume further that $\left(\|S\|_{1} \phi\right)^{n}(t) \rightarrow 0$ as $n \rightarrow \infty$ for each $t>0$. Then there exists a unique mild solution $u(n)$ of (4.1) such that $\lim _{|n| \rightarrow \infty} \frac{\|u(n)\|}{h(n)}=$ 0 . 
Proof Define the operator $F$ as in (4.2), then by hypothesis (F3), we have that

$$
\begin{aligned}
\|(F u)(n)\| & \leq \sum_{k=-\infty}^{n-1}\|S(n-1-k)\| \phi\left(\frac{\|u(n)\|}{h(n)}\right)+\|S\|_{1} \sup _{k \in \mathbb{Z}}\|f(k, 0)\| \\
& \leq\|S\|_{1}\left(\phi\left(\|u\|_{h}\right)+\sup _{k \in \mathbb{Z}}\|f(k, 0)\|\right) .
\end{aligned}
$$

Thus, $F$ is well defined. For $u, v \in \mathcal{C}_{h}^{0}(\mathbb{Z}, X)$, we have

$$
\|(F u)(n)-(F v)(n)\| \leq \sum_{k=-\infty}^{n-1}\|S(n-1-k)\| \phi\left(\frac{u(k)-v(k)}{h(k)}\right) \leq\|S\|_{1} \phi\left(\|u-v\|_{h}\right) .
$$

Therefore, $\|F u-F v\|_{h} \leq\|S\|_{1} \phi\left(\|u-v\|_{h}\right)$. Since $\left(\|S\|_{1} \phi\right)^{n}(t) \rightarrow 0$ as $n \rightarrow \infty$ for each $t>0$, by Matkowski's fixed point theorem, $F$ has a unique fixed point $u \in \mathcal{C}_{h}^{0}(\mathbb{Z}, X)$, so there exists a unique mild solution $u(n)$ of (4.1) such that $\lim _{|n| \rightarrow \infty} \frac{\|u(n)\|}{h(n)}=0$.

In the next theorem, the main tool used in proving the existence of solutions of (4.1) is the classical Leray-Schauder alternative theorem combined with Lemma 2.1.

Theorem 4.6 Suppose (H2), (F5). Then under the following additional condition: (A1) For all $a, b \in \mathbb{Z}, a \leq b$, and $\sigma>0$, the set $\{f(k, x): a \leq k \leq b,\|x\| \leq \sigma\}$ is relatively compact in $X$; there exists a function $h: \mathbb{Z} \rightarrow[1, \infty)$ such that $h(n) \rightarrow \infty$ as $|n| \rightarrow \infty$ and a mild solution $u(n)$ of (4.1) such that $\lim _{|n| \rightarrow \infty} \frac{\|u(n)\|}{h(n)}=0$.

Proof Define the function $h: \mathbb{Z} \rightarrow \mathbb{R}^{+}$by $h(k):=\left(\frac{L_{\infty}}{L_{f}(k)}\right)^{1 / \theta}$, where $L_{\infty}=\sup _{k \in \mathbb{Z}} L_{f}(k), k \in \mathbb{Z}$. Note that $h(k) \geq 1$ for all $k \in \mathbb{Z}$, and since $\lim _{|k| \rightarrow \infty} L_{f}(k)=0$, we also have $\lim _{|k| \rightarrow \infty} h(k)=$ $\infty$. Consider the operator $F$ as defined in (4.2). Using the Leray-Schauder alternative theorem, we will show that $F$ has a fixed point in $\mathcal{C}_{h}^{0}(\mathbb{Z}, X)$. The proof will be carried out in several steps.

(i) $F$ is well defined. For $u \in \mathcal{C}_{h}^{0}(\mathbb{Z}, X)$, one has

$$
\begin{aligned}
\|(F u)(n)\| & \leq \sum_{k=-\infty}^{n-1}\|S(n-1-k)\|\left(L_{f}(k)\|u(k)\|^{\theta}+\|f(k, 0)\|\right) \\
& \leq\|S\|_{1}\left(L_{\infty}\|u\|_{h}^{\theta}+\sup _{k \in \mathbb{Z}}\|f(k, 0)\|\right),
\end{aligned}
$$

whence $\lim _{|n| \rightarrow \infty} \frac{\|(F u)(n)\|}{h(n)}=0$. Thus $F$ is $\mathcal{C}_{h}^{0}(\mathbb{Z}, X)$-valued.

(ii) $F: \mathcal{C}_{h}^{0}(\mathbb{Z}, X) \rightarrow \mathcal{C}_{h}^{0}(\mathbb{Z}, X)$ is a continuous map. In fact, for $u, v \in \mathcal{C}_{h}^{0}(\mathbb{Z}, X)$, one has

$$
\begin{aligned}
& \|(F u)(n)-(F v)(n)\| \\
& \quad \leq \sum_{k=-\infty}^{n-1}\|S(n-1-k)\| L_{f}(k) h(k)^{\theta}\left(\frac{\|u(k)-v(k)\|}{h(k)}\right)^{\theta} \\
& \quad \leq L_{\infty}\|S\|_{1}\|u-v\|_{h}^{\theta} .
\end{aligned}
$$


Since that $h(k)=\left(\frac{L_{\infty}}{L_{f}(k)}\right)^{1 / \theta} \geq 1$, for all $k \in \mathbb{Z}$, then

$$
\sup _{n \in \mathbb{Z}} \frac{\|(F u)(n)-(F v)(n)\|}{h(n)} \leq L_{\infty}\|S\|_{1}\|u-v\|_{h}^{\theta} .
$$

So $\|F u-F v\|_{h} \leq L_{\infty}\|S\|_{1}\|u-v\|_{h}^{\theta}$. Hence $F$ is a continuous map.

(iii) $F$ is a completely continuous map. Let $r$ be a positive real number and $B_{r}(Z)$ be a closed ball with center at 0 and radius $r$ in the space $Z$. We set $V=F\left(B_{r}\left(\mathcal{C}_{h}^{0}(\mathbb{Z}, X)\right)\right)$ and $v=F u$ for $u \in B_{r}\left(\mathcal{C}_{h}^{0}(\mathbb{Z}, X)\right)$. We prove that, for each $n \in \mathbb{Z}$,

$$
\Omega_{n}(V):=\left\{\frac{v(n)}{h(n)}: v \in V\right\}
$$

is relatively compact in $X$. Indeed, for $\epsilon>0$, we choose $l \in \mathbb{Z}^{+}$such that

$$
\sum_{k=l}^{\infty}\|S(k)\|\left(L_{\infty} r^{\theta}+\sup _{k \in \mathbb{Z}}\|f(k, 0)\|\right) \leq \epsilon
$$

Since $v=F u$ for $u \in B_{r}\left(\mathbb{C}_{h}^{0}(\mathbb{Z}, X)\right)$,

$$
v(n)=\sum_{k=0}^{l-1} S(k) f(n-1-k, u(n-1-k))+\sum_{k=l}^{\infty} S(k) f(n-1-k, u(n-1-k)) .
$$

Thus,

$$
\begin{aligned}
\frac{v(n)}{h(n)}= & \frac{l}{h(n)}\left(\frac{1}{l} \sum_{k=0}^{l-1} S(k) f(n-1-k, u(n-1-k))\right) \\
& +\frac{1}{h(n)} \sum_{k=l}^{\infty} S(k) f(n-1-k, u(n-1-k)) .
\end{aligned}
$$

Note that

$$
\begin{aligned}
& \frac{1}{h(n)}\left\|\sum_{k=l}^{\infty} S(k) f(n-1-k, u(n-1-k))\right\| \\
& \quad \leq \frac{1}{h(n)} \sum_{k=l}^{\infty}\|S(k)\|\left(L_{\infty} r^{\theta}+\sup _{k \in \mathbb{Z}}\|f(k, 0)\|\right) \leq \epsilon .
\end{aligned}
$$

Therefore

$$
\frac{v(n)}{h(n)} \in \frac{l}{h(n)} \overline{\operatorname{co}(K)}+B_{\epsilon}(X)
$$

where $\overline{\operatorname{co}(K)}$ denotes the closed convex hull of $K$ and

$$
K=\bigcup_{k=0}^{l-1}\{S(k) f(\xi, x): \xi \in[n-l, n-1] \cap \mathbb{Z},\|x\| \leq R\}
$$


where $R=r \max \{h(\xi): \xi \in[n-l, n-1] \cap \mathbb{Z}\}$. Hence, $K$ is relatively compact since $S(k) \in \mathcal{B}(X)$ for all $k \in \mathbb{N}_{0}$, and $\{f(k, x): a \leq k \leq b,\|x\| \leq \sigma\}$ is relatively compact in $X$ for all $a, b \in \mathbb{Z}, a \leq b$, and $\sigma>0$. In view of

$$
\Omega_{n}(V) \subseteq \frac{l}{h(n)} \overline{\operatorname{co}(K)}+B_{\epsilon}(X),
$$

we deduce that $\Omega_{n}(V)$ is relatively compact in $X$.

Next, we prove that $V$ is weighted equiconvergent at $\pm \infty$. Indeed, proceeding as in (i), we have

$$
\begin{aligned}
\frac{\|v(n)\|}{h(n)} & \leq \frac{1}{h(n)} \sum_{k=-\infty}^{n-1}\|S(n-1-k)\|\left(L_{\infty}\|u\|_{h}^{\theta}+\|f(k, 0)\|\right) \\
& \leq \frac{1}{h(n)}\|S\|_{1}\left(L_{\infty} r^{\theta}+\sup _{k \in \mathbb{Z}}\|f(k, 0)\|\right),
\end{aligned}
$$

therefore $\frac{\|v(n)\|}{h(n)} \rightarrow 0$ as $|n| \rightarrow \infty$, and this convergence is independent of $u \in B_{r}\left(\mathcal{C}_{h}^{0}(\mathbb{Z}, X)\right)$. Hence $V$ satisfies the conditions of Lemma 2.1, so $V$ is a relatively compact set in $\mathcal{C}_{h}^{0}(\mathbb{Z}, X)$.

(iv) Now, we will prove that the set $\mathcal{C}:=\left\{u \in \mathcal{C}_{h}^{0}(\mathbb{Z}, X): u=\lambda F u, \lambda \in(0,1)\right\}$ is bounded. In fact, if $u \in \mathcal{C}_{h}^{0}(\mathbb{Z}, X)$ is a solution of $u=\lambda F u$ for $0<\lambda<1$, then

$$
\begin{aligned}
\frac{\|u(n)\|}{h(n)} & \leq \sum_{k=-\infty}^{n-1}\|S(n-1-k)\|\left(L_{\infty}\|u\|_{h}^{\theta}+\sup _{k \in \mathbb{Z}}\|f(k, 0)\|\right) \\
& \leq\|S\|_{1}\left(L_{\infty}\|u\|_{h}^{\theta}+\sup _{k \in \mathbb{Z}}\|f(k, 0)\|\right) .
\end{aligned}
$$

Hence

$$
\frac{\|u\|_{h}}{\|S\|_{1}\left(L_{\infty}\|u\|_{h}^{\theta}+\sup _{k \in \mathbb{Z}}\|f(k, 0)\|\right)} \leq 1 .
$$

Observe that in view of the condition $\theta<1$, it follows that $\mathcal{C}$ is bounded.

(v) There exists $r_{0}>0$ such that $F\left(B_{r_{0}}\left(\mathcal{C}_{h}^{0}(\mathbb{Z}, X)\right)\right) \subset B_{r_{0}}\left(\mathcal{C}_{h}^{0}(\mathbb{Z}, X)\right)$. Assume that the assertion is false, then for all $r>0$, arguing similarly as in (iv), we deduce that $1 \leq\|S\|_{1}\left(L_{\infty} r^{\theta-1}+\frac{1}{r} \sup _{k \in \mathbb{Z}}\|f(k, 0)\|\right)$, which is a contradiction because $\theta<1$.

(vi) Finally, by Theorem 2.3, $F$ has a fixed point $u \in \mathcal{C}_{h}^{0}(\mathbb{Z}, X)$.

In the next results, we study the existence and uniqueness of $W P A A_{d}$ and $W P S A P_{\omega}$ mild solutions of (4.1).

Theorem 4.7 Let $\rho_{1}, \rho_{2} \in V_{\infty}$ and $\rho \in U_{T}$ be given. Assume that (H2) holds, $f \in \Omega \in$ $\mathcal{M}(\mathbb{Z} \times X, X)$ is globally Lipschitz in the following sense:

$$
\|f(n, x)-f(n, y)\| \leq L\|x-y\| \quad \text { for all } n \in \mathbb{Z} \text { and all } x, y \in X
$$

where $L<\frac{1}{\|S\|_{1}}$, then (4.1) has a unique mild solution $u$ which belongs to the corresponding subset $\Omega \in \mathcal{M}(\mathbb{Z}, X)$. 
Proof Consider the operator $F: W P A A_{d}(\mathbb{Z}, X) \rightarrow W P A A_{d}(\mathbb{Z}, X)$ defined by

$$
(F u)(n):=\sum_{k=-\infty}^{n-1} S(n-1-k) f(k, u(k)), \quad n \in \mathbb{Z}
$$

where $f \in W P A A_{d}(\mathbb{Z}, X)$. Note that $F$ is well defined by [33, Theorem 3.1] and Theorem 3.17. In addition, for $u, v \in W P A A_{d}(\mathbb{Z}, X)$ and $n \in \mathbb{Z}$, the following inequality holds:

$$
\|(F u)(n)-(F v)(n)\|=\left\|\sum_{k=-\infty}^{n-1} S(n-1-k)(f(k, u(k))-f(k, v(k)))\right\| \leq L\|S\|_{1}\|u-v\|_{\infty} .
$$

Since $\|S\|_{1} L<1$, we conclude that $F$ is a contraction, and using the Banach fixed point theorem we get that there exists a unique discrete weighted pseudo almost automorphic solution of (4.1). The proof for the space of S-asymptotic $\omega$-periodic sequences is analogous, but in this case, we use [34, Theorem 2.3] and Theorem 3.17 in order to prove that $F$ is well defined.

In the following theorem, we show that with a local Lipschitz condition on $f$ the conclusion of the previous theorem holds.

Theorem 4.8 Let $\rho_{1}, \rho_{2} \in V_{\infty}$ and $\rho$ in $U_{T}$ be given. Assume that (H2) holds, and let $f \in$ $\mathcal{M}(\mathbb{Z} \times X, X)$ that satisfies a local Lipschitz condition. If there exists $r_{0}>0$ such that

$$
\|S\|_{1}\left(L\left(r_{0}\right)+\frac{\sup _{k \in \mathbb{Z}}\|f(k, 0)\|}{r_{0}}\right)<1,
$$

then the semilinear difference equation (4.1) has a unique mild solution $u$ which belongs to the same space as $f$ with $\|u\|_{\infty} \leq r_{0}$.

Proof Consider $f \in W P A A_{d}(\mathbb{Z} \times X, X)$. Note that $F: W P A A_{d}(\mathbb{Z}, X) \rightarrow W P A A_{d}(\mathbb{Z}, X)$ given by (4.4) is well defined by Corollary [5, Corollary 2.4] and Theorem 3.17.

Let

$$
B_{r_{0}}(0):=\left\{u \in W P A A_{d}(\mathbb{Z}, X):\|u\|_{\infty}<r_{0}\right\}
$$

We show that $F\left(B_{r_{0}}(0)\right) \subset B_{r_{0}}(0)$. Indeed, let $u$ be in $B_{r_{0}}(0)$. Since $f$ is locally Lipschitz, we obtain

$$
\|f(k, u(k))\| \leq\|f(k, u(k))-f(k, 0)\|+\|f(k, 0)\| \leq L\left(r_{0}\right)\|u(k)\|+\|f(k, 0)\| \quad \text { for } k \in \mathbb{Z} .
$$

Moreover, we have the estimate

$$
\begin{aligned}
\|F(u)(n)\| & \leq \sum_{k=-\infty}^{n-1}\|S(n-1-k)\| \|\left(f(k, u(k))-f(k, 0)\left\|+\sum_{k=-\infty}^{n-1}\right\| S(n-1-k) f(k, 0) \|\right. \\
& \leq L\left(r_{0}\right) \sum_{k=-\infty}^{n-1}\|S(n-1-k)\|\|u(k)\|+\|S\|_{1} \sup _{k}\|f(k, 0)\|
\end{aligned}
$$




$$
\leq\|S\|_{1}\left(L\left(r_{0}\right)+\frac{\sup _{k}\|f(k, 0)\|}{r_{0}}\right) r_{0} \leq r_{0}
$$

proving the claim. On the other hand, for $u, v \in B_{r_{0}}(0)$, we have that

$$
\begin{aligned}
\|F u(n)-F v(n)\| & \leq \sum_{k=-\infty}^{n-1}\|S(n-1-k)\|\|(f(k, u(k))-f(k, v(k)))\| \\
& \leq L\left(r_{0}\right) \sum_{k=-\infty}^{n-1}\|S(n-1-k)\|\|u(k)-v(k)\| \leq\|S\|_{1} L\left(r_{0}\right)\|u-v\|_{\infty} .
\end{aligned}
$$

Observing that $\|S\|_{1} L\left(r_{0}\right)<1$, it follows that $F$ is a contraction in $B_{r_{0}}(0)$. Then there is a unique $u \in B_{r_{0}}(0)$ such that $F u=u$. Analogously, we can prove the theorem for $f \in \operatorname{WPSAP}_{\omega}(\mathbb{Z} \times X, X, \rho)$. For that purpose, we use [34, Theorem 2.3] and Theorem 3.17 in order to prove that $F$ is well defined, and we just have to take the ball of radius $r_{0}$ in $\operatorname{WPSAP}_{\omega}(\mathbb{Z} \times X, X, \rho)$.

Theorem 4.9 Let $\rho_{1}, \rho_{2} \in V_{\infty}$ and $\rho \in U_{T}$ be given. Assume that $(\mathrm{H} 2)$ holds and $f \in \Omega \in$ $\mathcal{M}(\mathbb{Z} \times X, X)$ satisfies the following condition:

$$
\|f(n, x)-f(n, y)\| \leq \phi(\|x-y\|) \quad \text { for all } n \in \mathbb{Z} \text { and all } x, y \in X,
$$

where $\phi: \mathbb{R}^{+} \rightarrow \mathbb{R}^{+}$is a nondecreasing function. Then (4.1) has a unique mild solution $u$ which belongs to the corresponding subset $\Omega \in \mathcal{M}(\mathbb{Z}, X)$, provided $(\|S\| \phi)^{n}(t) \rightarrow 0$ as $n \rightarrow \infty$ for each $t>0$.

Proof Consider the operator $F: W P A A_{d}(\mathbb{Z}, X) \rightarrow W P A A_{d}(\mathbb{Z}, X)$ defined by

$$
(F u)(n):=\sum_{k=-\infty}^{n-1} S(n-1-k) f(k, u(k)), \quad n \in \mathbb{Z}
$$

where $f \in W P A A_{d}(\mathbb{Z}, X)$. Note that $F$ is well defined by [34, Theorem 2.3] and Theorem 3.17. In addition, for $u, v \in W P A A_{d}(\mathbb{Z}, X)$ and $n \in \mathbb{Z}$, the following inequality holds:

$$
\|(F u)(n)-(F v)(n)\|=\left\|\sum_{k=-\infty}^{n-1}\right\| S(n-1-k)(f(k, u(k))-f(k, v(k)))\|\leq L\| S \|_{1} \phi(\|u-v\|) .
$$

Since $\left(\|S\|_{1} \phi\right)^{n}(t) \rightarrow 0$ as $n \rightarrow \infty$ for each $t>0$, by Matkowski's fixed point theorem (Theorem 2.2), $F$ has a unique fixed point $u \in W P A A_{d}(\mathbb{Z}, X)$, so there is a unique mild solution $u(n)$ of (4.1). The proof for the space of S-asymptotic $\omega$-periodic sequences is analogous, we just use Theorem [34, Theorem 2.3] and Theorem 3.17 in order to prove that $F$ is well defined.

In the following theorem, we study under certain non-Lipschitz conditions on the function $f$ the existence of solutions in $\operatorname{WPSAP}_{\omega}(Z \times X, X)$ of equation (4.1). We consider functions $f: \mathbb{Z} \times X \rightarrow X$ to establish our result. 
Remark 4.10 Hypothesis (B1) has also been considered previously in [3] and [34] in order to prove the existence of mild solutions for a class of semilinear difference equations in a Banach space $X$.

Theorem 4.11 Assume that $f \in \operatorname{WPSAP}_{\omega}(Z \times X, X) \cap \mathcal{U} \mathcal{C}_{k}(\mathbb{Z} \times X, X), \rho_{1}, \rho_{2} \in V_{\infty}$ and that the assumptions (H1), (H2), and (A1) hold. Under the following additional conditions:

(B1) There exist a nondecreasing function $W: \mathbb{R}^{+} \rightarrow \mathbb{R}^{+}$and a function $M: \mathbb{Z} \rightarrow \mathbb{R}^{+}$ such that $\|f(k, x)\| \leq M(k) W(\|x\|)$ for all $k \in \mathbb{Z}, x \in X$.

(B2) For each $v>0, \lim _{|n| \rightarrow \infty} \frac{1}{h(n)} \sum_{k=-\infty}^{n-1}\|S(n-k-1)\| M(k) W(v h(k))=0$, where $h$ is given in Lemma 2.1.

(B3) For each $\epsilon>0$, there exists $\delta>0$ such that, for $u, v \in \mathcal{C}_{h}^{0}(\mathbb{Z}, X),\|u-v\|_{h} \leq \delta$ implies that $\sum_{k=-\infty}^{n}\|S(n-k)\| \| f(k, u(k)-f(k, v(k)) \| \leq \epsilon$ for all $n \in \mathbb{Z}$.

(B4) $\liminf _{r \rightarrow \infty} \frac{r}{\beta(r)}>1$, where

$$
\beta(r)=\sup _{n \in \mathbb{Z}}\left(\frac{1}{h(n+1)} \sum_{k=-\infty}^{n}\|S(n-k)\| M(k) W(r h(k))\right) .
$$

Then (4.1) has a mild solution in $\operatorname{WPSAP}_{\omega}(Z \times X, X)$.

Proof Consider the operator $F: \mathcal{C}_{h}^{0}(\mathbb{Z}, X) \rightarrow \mathcal{C}_{h}^{0}(\mathbb{Z}, X)$ defined by

$$
(F u)(n):=\sum_{k=-\infty}^{n-1} S(n-1-k) f(k, u(k)), \quad n \in \mathbb{Z}
$$

by Leray-Schauder theorem, we will prove that $F$ has a fixed point in $\operatorname{WPSAP}_{\omega}(Z \times X, X)$. We divide the proof into several steps.

(i) $F$ is well defined. For $u \in \mathcal{C}_{h}^{0}(\mathbb{Z}, X)$, by (B1), one has

$$
\begin{aligned}
\|(F u)(n)\| & \leq \sum_{k=-\infty}^{n-1}\|S(n-1-k)\| M(k) W(\|u(k)\|) \\
& \leq \sum_{k=-\infty}^{n-1}\|S(n-1-k)\| M(k) W\left(\|u\|_{h} h(k)\right),
\end{aligned}
$$

whence $\frac{\|(F u)(n)\|}{h(n)} \leq \frac{1}{h(n)} \sum_{k=-\infty}^{n-1}\|S(n-1-k)\| M(k) W\left(\|u\|_{h} h(k)\right)$. It follows from (B2) that $F$ is $\mathcal{C}_{h}^{0}(\mathbb{Z}, X)$-valued.

(ii) $F$ is continuous. In fact, for each $\epsilon>0$, by (B3) there exists $\delta>0$ such that, for $u, v \in \mathcal{C}_{h}^{0}(\mathbb{Z}, X),\|u-v\|_{h} \leq \delta$, one has

$$
\|(F u)(n)-(F v)(n)\| \leq \sum_{k=-\infty}^{n-1}\|S(n-1-k)\| \| f(k, u(k)-f(k, v(k)) \|,
$$

since $h(n) \geq 1$, by (B3), one has $\|F u-F v\|_{h} \leq \epsilon$, hence $F$ is continuous.

(iii) $F$ is completely continuous. Let $V=F\left(B_{r}\left(\mathcal{C}_{h}^{0}(\mathbb{Z}, X)\right)\right)$ and $v=F u$ for $u \in \mathcal{C}_{h}^{0}(\mathbb{Z}, X)$. First, we prove that $\Omega_{n}(V):=\left\{\frac{v(n)}{h(n)}: v \in V\right\}$ is relatively compact in $X$ for each 
$n \in \mathbb{Z}$. By (B2), for $\epsilon>0$, we can choose $l \in \mathbb{Z}^{+}$such that

$$
\sum_{k=l}^{\infty}\|S(k)\| M(n-1-k) W(\operatorname{rh}(n-1-k)) \leq \epsilon
$$

Since $v=F u$ for $u \in \mathcal{C}_{h}^{0}(\mathbb{Z}, X)$, then

$$
\begin{aligned}
\frac{v(n)}{h(n)}= & \frac{l}{h(n)}\left(\frac{1}{l} \sum_{k=0}^{l-1} S(k) f(n-1-k, u(n-1-k))\right) \\
& +\frac{1}{h(n)} \sum_{k=l}^{\infty} S(k) f(n-1-k, u(n-1-k)) .
\end{aligned}
$$

Note that

$$
\begin{aligned}
& \frac{1}{h(n)}\left\|\sum_{k=l}^{\infty} S(k) f(n-1-k, u(n-1-k))\right\| \\
& \quad \leq \frac{1}{h(n)} \sum_{k=l}^{\infty}\|S(k)\| M(n-1-k) W(r h(n-1-k)) \| \leq \epsilon .
\end{aligned}
$$

So (4.3) holds. Then $\Omega_{n}(V)$ is relatively compact in $X$ for all $n \in \mathbb{Z}$. Next, we show that $V$ is weighted equiconvergent at $\pm \infty$. In fact,

$$
\frac{\|v(n)\|}{h(n)} \leq \frac{1}{h(n)} \sum_{k=-\infty}^{n-1}\|S(n-1-k)\| M(k) W(r h(k))
$$

hence $\frac{\|v(n)\|}{h(n)} \rightarrow 0$ as $|n| \rightarrow \infty$ by (B2), and this convergence is independent of $u \in B_{r}\left(\mathcal{C}_{h}^{0}(\mathbb{Z}, X)\right), V$ is a relatively compact set in $\mathcal{C}_{h}^{0}(\mathbb{Z}, X)$ by Lemma 2.1 .

(iv) Now, we will prove that the set

$$
\left\{u \in \mathcal{C}_{h}^{0}(\mathbb{Z}, X): u=\lambda F u, \lambda \in(0,1)\right\}
$$

is bounded. In fact, if $u \in \mathcal{C}_{h}^{0}(\mathbb{Z}, X)$ is a solution of $u=\lambda F u$ for $0<\lambda<1$, then

$$
\|u(n)\| \leq \sum_{k=-\infty}^{n-1}\|S(n-1-k)\| M(k) W\left(\|u\|_{h} h(k)\right) \leq h(n) \beta\left(\|u\|_{h}\right)
$$

Hence, $\frac{\|u\|_{h}}{\beta\left(\|u\|_{h}\right)} \leq 1$. We conclude using (B4).

(v) There exists $r_{0}>0$ such that $F\left(B_{r_{0}}\left(\mathcal{C}_{h}^{0}(\mathbb{Z}, X)\right)\right) \subset B_{r_{0}}\left(\mathcal{C}_{h}^{0}(\mathbb{Z}, X)\right)$. Assume that the assertion is false, then for all $r>0$, we can choose $u^{r} \in B_{r_{0}}\left(\mathcal{C}_{h}^{0}(\mathbb{Z}, X)\right)$ such that $\left\|F u^{r}\right\|_{h}>r$. Similar as (iv), we deduce that $\frac{r}{\beta(r)} \leq 1$, then $\liminf _{\xi \rightarrow \infty} \frac{\xi}{\beta(\xi)} \leq 1$, which contradicts condition (B4).

(vi) It follows from Theorem [34, Theorem 2.3] and Theorem 3.17, that the vector-valued space $\operatorname{WPSAP}_{\omega}(\mathbb{Z}, X)$ is invariant under $F$. Consequently, combining this with step (iv), we have that

$$
F\left(B_{r_{0}}\left(\mathcal{C}_{h}^{0}(\mathbb{Z}, X)\right) \cap \operatorname{WPSAP}_{\omega}(\mathbb{Z}, X)\right) \subseteq B_{r_{0}}\left(\mathcal{C}_{h}^{0}(\mathbb{Z}, X)\right) \cap \operatorname{WPSAP}_{\omega}(\mathbb{Z}, X)
$$


Hence, we arrive at the following conclusion:

$$
\begin{aligned}
& F\left(\overline{B_{r_{0}}\left(\mathcal{C}_{h}^{0}(\mathbb{Z}, X)\right) \cap \operatorname{WPSAP}_{\omega}(\mathbb{Z}, X)}{ }^{C_{h}^{0}(\mathbb{Z}, X)}\right) \\
& \subseteq \overline{F\left(B_{r_{0}}\left(\mathcal{C}_{h}^{0}(\mathbb{Z}, X)\right) \cap \operatorname{WPSAP}_{\omega}(\mathbb{Z}, X)\right)} \mathcal{C}_{h}^{0},(\mathbb{Z}, X) \\
& \subseteq{\overline{B_{r_{0}}\left(\mathcal{C}_{h}^{0}(\mathbb{Z}, X)\right) \cap \operatorname{WPSAP}_{\omega}(\mathbb{Z}, X)}}_{\mathcal{C}_{h}^{0},(\mathbb{Z}, X)},
\end{aligned}
$$

where $\bar{B}_{h}^{0}(\mathbb{Z}, X)$ denotes the closure of a set $B$ in the space $\mathcal{C}_{h}^{0}(\mathbb{Z}, X)$. Thus, we can consider the following application:

$$
F: \overline{B_{r_{0}}\left(\mathcal{C}_{h}^{0}(\mathbb{Z}, X)\right) \cap \operatorname{WPSAP}_{\omega}(\mathbb{Z}, X)}{ }_{h}^{\mathcal{C}_{h}^{0}(\mathbb{Z}, X)} \rightarrow \overline{B_{r_{0}}\left(\mathcal{C}_{h}^{0}(\mathbb{Z}, X)\right) \cap \operatorname{WPSAP}_{\omega}(\mathbb{Z}, X)}{ }_{h}^{\mathcal{C}_{h}^{0}(\mathbb{Z}, X)}
$$

By (i)-(iii), we have that $F$ is completely continuous. Applying (iv) and the Leray-Schauder theorem (Theorem 2.3), $F$ has a fixed point $u$ which belongs to the space ${\overline{B_{r_{0}}\left(\mathcal{C}_{h}^{0}(\mathbb{Z}, X)\right) \cap W_{P S A P}(\mathbb{Z}, X)}}^{\mathcal{C}_{h}^{0}(\mathbb{Z}, X)}$.

(vii) Finally, we prove that $u$ (the fixed point of $F$ given in (vi)) is discrete weighted pseudo S-asymptotically $\omega$-periodic. Indeed, let $\left(u_{m}\right)_{m}$ be a sequence in $B_{r_{0}}\left(\mathcal{C}_{h}^{0}(\mathbb{Z}, X)\right) \cap \operatorname{WPSAP}_{\omega}(\mathbb{Z}, X)$ such that $u_{m} \rightarrow u$, as $m \rightarrow \infty$ in the norm $\mathcal{C}_{h}^{0}(\mathbb{Z}, X)$. For $\epsilon>0$, let $\delta>0$ be the constant in (B3), that is, there exists $m_{0} \in \mathbb{Z}^{+}$ such that $\left\|u_{m}-u\right\|_{h} \leq \delta$ for all $m \geq m_{0}$. Note that for $m \geq m_{0}$,

$$
\left\|F u_{m}-F u\right\|_{\infty} \leq \sup _{n \in \mathbb{Z}} \sum_{k=-\infty}^{n-1}\|S(n-1-k)\|\left\|f\left(k, u_{m}(k)\right)-f(k \cdot u(k))\right\| \leq \epsilon,
$$

which implies that $\left(F u_{m}\right)_{m}$ converges to $F u=u$ uniformly in $\mathbb{Z}$. Since $F u_{m}$ belongs to $\operatorname{WPSAP}_{\omega}(\mathbb{Z}, X)$, we get that $u \in \operatorname{WPSAP}_{\omega}(\mathbb{Z}, X)$.

Theorem 4.12 Assume that $f \in \operatorname{WPSAP}_{\omega}(Z \times X, X), \rho_{1}, \rho_{2} \in V_{\infty}$, (H2), (A1) hold and the following conditions are satisfied:

(B5) There exists a nondecreasing and surjective function $\mathcal{W}: \mathbb{R}^{+} \rightarrow \mathbb{R}^{+}$such that

$$
\| f(k, h(k) u)-f(k, h(k) v \| \leq \mathcal{W}(\|u-v\|) \quad \text { for all } k \in \mathbb{Z}, u, v \in X,
$$

where $h$ is given in Lemma 2.1.

(B6) $\liminf _{\tau \rightarrow \infty} \frac{\tau}{\|S\|_{1}\left(\mathcal{W}(\tau)+\sup _{k \in \mathbb{Z}}\|f(k, 0)\|\right)}>1$.

Then (3.1) has a mild solution $u \in \operatorname{WPSAP}_{\omega}(\mathbb{Z}, X)$.

Proof Consider the operator $F: \mathcal{C}_{h}^{0}(\mathbb{Z}, X) \rightarrow \mathcal{C}_{h}^{0}(\mathbb{Z}, X)$ defined by

$$
(F u)(n):=\sum_{k=-\infty}^{n-1} S(n-1-k) f(k, u(k)), \quad n \in \mathbb{Z}
$$

For $u, v \in \mathcal{C}_{h}^{0}(\mathbb{Z}, X)$, we have the following estimates:

$$
\|F u(n)\| \leq \sum_{k=-\infty}^{n-1}\|S(n-1-k)\|\|f(k, u(k))\| \leq\|S\|_{1}\left(\mathcal{W}\left(\|u\|_{h}\right)+\sup _{k \in \mathbb{Z}}\|f(k, 0)\|\right),
$$


and $\|f(k, u)-f(k, v)\| \leq \mathcal{W}\left(\frac{\|u-v\|}{h(k)}\right) \leq \mathcal{W}(\|u-v\|)$, hence $F$ is well defined and $f \in \mathcal{U C}(\mathbb{Z} \times$ $X, X)$. For $u, v \in \mathcal{C}_{h}^{0}(\mathbb{Z}, X)$, we have

$$
\|(F u(n))-(F v(n))\| \leq \sum_{k=-\infty}^{n-1}\|S(n-1-k)\| \mathcal{W}\left(\frac{\|u(k)-v(k)\|}{h(k)}\right) \leq\|S\|_{1} \mathcal{W}\left(\|u-v\|_{h}\right),
$$

which implies that $F$ is continuous. Next, let $V=F\left(B_{r}\left(\mathcal{C}_{h}^{0}(\mathbb{Z}, X)\right)\right)$ and $v=F u$ for $u \in$ $B_{r}\left(\mathcal{C}_{h}^{0}(\mathbb{Z}, X)\right)$. For $\epsilon>0$, we choose $l \in \mathbb{Z}^{+}$such that $\sum_{k=l}^{\infty}\|S(k)\|\left(\mathcal{W}(r)+\sup _{k \in \mathbb{Z}}\|f(k, 0)\|\right) \leq$ $\epsilon$. Let $u \in B_{r}\left(\mathcal{C}_{h}^{0}(\mathbb{Z}, X)\right)$, we have

$$
\begin{aligned}
& \frac{1}{h(n)} \| \sum_{k=l}^{\infty} S(k) f(n-1-k, u(n-1-k)) \| \\
& \quad \leq \sum_{k=l}^{\infty}\|S(k)\|\left(\mathcal{W}(r)+\sup _{k \in \mathbb{Z}}\|f(k, 0)\|\right) \leq \epsilon .
\end{aligned}
$$

From (4.3), (4.7), and (A1), we have that $\Omega_{n}(V)$ is relatively compact in $X$ for all $n \in \mathbb{Z}$. For $u \in B_{r}\left(\mathcal{C}_{h}^{0}(\mathbb{Z}, X)\right)$, by (4.6), we have

$$
\frac{\|v(n)\|}{h(n)} \leq \frac{\|S\|_{1}}{h(n)}\left(\mathcal{W}(r)+\sup _{k \in \mathbb{Z}}\|f(k, 0)\|\right)
$$

Hence $\lim _{|n| \rightarrow \infty} \frac{\|v(n)\|}{h(n)}=0$ uniformly in $u \in B_{r}\left(\mathcal{C}_{h}^{0}(\mathbb{Z}, X)\right)$. By Lemma 2.1, $F$ is completely continuous.

Finally, we prove the boundedness of the set defined in (4.5). If $u \in B_{r}\left(\mathcal{C}_{h}^{0}(\mathbb{Z}, X)\right)$ is a solution of $u=\lambda F u$ for $0<\lambda<1$, then by (4.6)

$$
\frac{\|u\|_{h}}{\|S\|_{1}\left(\mathcal{W}\left(\|u\|_{h}\right)+\sup _{k \in \mathbb{Z}}\|f(k, 0)\|\right)} \leq 1 .
$$

From (B6), we conclude that the set (4.5) is bounded. Similar as the proof of Theorem 4.11, (4.1) has a mild solution $u \in \operatorname{WPSAP}_{\omega}(\mathbb{Z}, X)$ by Theorem 2.3 .

\section{An example}

Let $a>0$. Define $a(n)=\frac{1}{a}\left(1-\frac{1}{(1+a)^{n+1}}\right)$ and $b(n)=1-\frac{n-1}{2^{n+2}}$. Then one can verify that $a(n)=$ $\int_{0}^{\infty} \rho_{n}(t) k(t) d t$ and $b(n)=\int_{0}^{\infty} \rho_{n}(t) g(t) d t$, where $k(t)=\int_{0}^{t} e^{-a s} d s$ and $g(t)=1-e^{-t}(t+1)$ in a straightforward way. Denote $a_{1}(t)=e^{-a t}, t \geq 0$, then $a_{1} \in L^{1}\left(\mathbb{R}_{+}\right), a_{1}(t) \geq 0$, and $-a_{1}^{\prime}(t) \geq 0$ for all $t>0$, nonincreasing and convex, so $a_{1}$ is 3-monotone. Thus $a_{1}(t)$ is 2-regular and of positive type by [31, Proposition 3.3]. It is easy to see that $k(t)$ is of positive type, and by the remarks following [31, Definition 3.3] it follows that $k(t)$ is 2-regular too.

Clearly $g(0)=0$ and $g \in W^{1,1}\left(\mathbb{R}_{+}\right)$. Note that

$$
\widehat{k}(\lambda)=\frac{a_{0}}{\lambda}+\frac{\widehat{a_{1}}(\lambda)}{\lambda} .
$$

By [31, p. 266] $\frac{1}{\lambda k(\lambda)}$ is locally analytic at $\lambda=0$. Thus $k(t)$ satisfies the hypotheses of Theorem 3.9. Therefore, $A$ generates a summable discrete resolvent family $\{R(n)\}_{n \in \mathbb{N}_{0}}$ with $a(n)=\frac{1}{a}\left(1-\frac{1}{(1+a)^{n+1}}\right)$ and $b(n)=1-\frac{n-1}{2^{n+2}}$ such that $\|R(n)\| \leq M, M>0$ for all $n \in \mathbb{N}$. We 
set $X=L^{2}([0,1])$, and we consider the second order differential operator $A z(\xi)=\partial_{\xi \xi}^{2} z(\xi)$ with domain $D(A)=\left\{z \in H^{2}[0,1]: z(0)=z(1)=0\right\}$. It is well known that $A$ generates a bounded analytic semigroup on $L^{2}[0,1]$ (see [18, Example 4.8]). Let us consider the following differential-difference Volterra equation on $X=L^{2}([0,1])$ :

$$
\begin{aligned}
u(n+1, x) & =\sum_{k=-\infty}^{n} a(n-k) \partial_{x x}^{2} u(k+1, x)+\sum_{k=-\infty}^{n} b(n-k) f(k, u(k, x)), \\
n \in \mathbb{Z}, x \in[0,1] &
\end{aligned}
$$

where $f(k, u)=\frac{\sin u}{e^{k^{2}} K}$, with $K>M$. Consider $h(n)=e^{n^{2}}$, then $h(n) \geq 1$ for all $n \in \mathbb{Z}$ and $\lim _{|n| \rightarrow \infty} h(n)=\infty$. On the other hand,

$$
\|f(k, h(k) u)-f(k, h(k) v)\|=\left\|\frac{\sin \left(e^{k^{2}} u\right)-\sin \left(e^{k^{2}} v\right)}{K e^{k^{2}}}\right\| \leq \frac{\|u-v\|}{K} .
$$

Therefore, (H2), (F1) hold and $L_{f}\|R\|_{1}<1$ with $L_{f}=1 / K$. Thus by Theorem 4.2 there is a unique mild solution $u(n)$ of (5.1) such that $\lim _{|n| \rightarrow \infty} \frac{\|u(n)\|}{h(n)}=0$.

\section{Acknowledgements}

Not applicable.

\section{Funding}

The work of VK and MW is partially supported by the Air Force Office of Scientific Research (AFOSR) under Award NO: FA9550-18-1-0242; CL was partially supported by FONDECYT, Grant No 1180041; SR was funded by the CONICYT-PFCHA/Doctorado Nacional/2017-21171405.

\section{Availability of data and materials}

Not applicable.

\section{Competing interests}

The authors declare that they have no competing interests.

\section{Authors' contributions}

The authors contributed equally and significantly in writing this paper. All authors read and approved the final manuscript.

\section{Author details}

'Department of Mathematics, Faculty of Natural Sciences, University of Puerto Rico, San Juan, Puerto Rico.

²Departamento de Matemática y Ciencia de la Computación, Facultad de Ciencias, Universidad de Santiago de Chile, Santiago, Chile.

\section{Publisher's Note}

Springer Nature remains neutral with regard to jurisdictional claims in published maps and institutional affiliations.

Received: 16 April 2019 Accepted: 12 June 2019 Published online: 27 June 2019

\section{References}

1. Abadias, L., Lizama, C.: Almost automorphic mild solutions to fractional partial difference-differential equations. Appl. Anal. 95(6), 1347-1369 (2016)

2. Abu-Saris, R., Al-Mdallal, Q: On the asymptotic stability of linear system of fractional-order difference equations. Fract. Calc. Appl. Anal. 16(3), 613-629 (2013)

3. Agarwal, R., Cuevas, C., Dantas, F.: Almost automorphy profile of solutions for difference equations of Volterra type. J. Appl. Math. Comput. 42, 1-18 (2013)

4. Agarwal, R.P., Cuevas, C., Lizama, C.: Regularity of Difference Equations on Banach Spaces. Springer, Cham (2014)

5. Alvarez, E., Lizama, C.: Weighted pseudo almost automorphic and S-asymptotically $\omega$-periodic solutions to fractional difference-differential equations. Electron. J. Differ. Equ. 2016, 270 (2016)

6. Araya, D., Castro, R., Lizama, C.: Almost automorphic solutions of difference equations. Adv. Differ. Equ. 2009, Article ID 591380 (2009)

7. Atici, F.M., Eloe, P.W.: Initial value problems in discrete fractional calculus. Proc. Am. Math. Soc. 137(3), 981-989 (2009) 
8. Bateman, H.: Some simple differential difference equations and the related functions. Bull. Am. Math. Soc. 49, 494-512 (1943)

9. Blunck, S.: Maximal regularity of discrete and continuous time evolution equations. Stud. Math. 146(2), 157-176 (2001)

10. Blunck, S.: Analyticity and discrete maximal regularity on $L_{p}$-spaces. J. Funct. Anal. 183(1), 211-230 (2001)

11. Boor, C., Höllig, K., Sherma, S.: Some simple differential difference equations and the related functions. Am. J. Math. 111(3), 403-415 (1989)

12. Braverman, H.E., Saker, S.H.: Permanence, oscillation and attractivity of the discrete hematopoiesis model with variable coefficients. Nonlinear Anal. 67, 2955-2965 (2007)

13. Chen, L., Meng, X.: Periodic solution and almost periodic solution for a nonautonomous Lotka-Volterra dispersal system with infinite delay. J. Math. Anal. Appl. 339, 125-145 (2008)

14. Cuevas, C., Lizama, C.: Semilinear evolution equation of second order via maximal regularity. Adv. Differ. Equ. 2008, $316207(2008)$

15. Cuevas, C., Pinto, M.: Convergent solutions of linear functional difference equations in phase space. J. Math. Anal. Appl. 277, 324-341 (2003)

16. Diamond, P.: Domains of stability and resilience for biological populations obeying difference equations. J. Theor. Biol. 61, 287-306 (1976)

17. Elaydi, S.: Stability and asymptoticity of Volterra difference equations: a progress report. J. Comput. Appl. Math. 228(2), 504-513 (2009)

18. Engel, K.J., Nagel, R.: One-Parameter Semigroups for Linear Evolution Equations. Springer, Berlin (2000)

19. Granas, A., Dugundji, J.: Fixed Point Theory. Springer, New York (2003)

20. Gronek, T., Schmeidel, E.: Existence of bounded solution of Volterra difference equations via Darbo's fixed-point theorem. J. Differ. Equ. Appl. 19(10), 1645-1653 (2013)

21. Kemmochi, T.: Discrete maximal regularity for abstract Cauchy problems. Stud. Math. 234(3), 241-263 (2016)

22. Kemmochi, T., Saito, N.: Discrete maximal regularity and the finite element method for parabolic equations. Numer. Math. 138(4), 905-937 (2018)

23. Kulenović, M.R.S., Nurkanović, M.: Asymptotic behavior of a system of linear fractional difference equations. J. Inequal. Appl. 2005, 741584 (2005)

24. Li, Y., Sun, D.: Microscopic car-following model for the traffic flow: the state of the art. J. Control Theory Appl. 10(2), 133-143 (2012)

25. Lizama, C.: $\ell_{p}$-maximal regularity for fractional difference equations on UMD spaces. Math. Nachr. 288(17/18), 2079-2092 (2015)

26. Lizama, C.: The Poisson distribution, abstract fractional difference equations, and stability. Proc. Am. Math. Soc. 145 3809-3827 (2017)

27. Lizama, C., Murillo, M.: Maximal regularity in $I_{p}$ spaces for discrete time fractional shifted equations. J. Differ. Equ. 263, 3175-3196 (2017)

28. Matkowski, J.: Integrable solutions of functional equations. Diss. Math. 127, 1-68 (1975)

29. Mazanov, A.: Acceptor control in model ecosystems. J. Theor. Biol. 71(1), $21-38$ (1978)

30. Nieto, J., Ouahab, A., Slimani, M.: Existence and boundedness of solutions for systems of difference equations with infinite delay. Glas. Mat. 53(73), 123-141 (2018)

31. Prüss, J.: Evolutionary Integral Equations and Applications. Monographs in Mathematics, vol. 87. Birkhäuser Verlag, Basel (1993)

32. Song, Y.: Positive almost periodic solutions of nonlinear discrete systems with finite delay. Comput. Math. Appl. 58, 128-134 (2009)

33. Xia, Z:: Discrete weighted pseudo-almost automorphy and applications. J. Appl. Math. 2014, 984946 (2014)

34. Xia, Z., Wang, D.: Asymptotic behavior of mild solutions for nonlinear fractional difference equations. Fract. Calc. Appl. Anal. 21(2), 527-551 (2018)

35. Yosida, K.: Functional Analysis. Springer, Berlin (1980)

\section{Submit your manuscript to a SpringerOpen ${ }^{\circ}$ journal and benefit from:}

- Convenient online submission

- Rigorous peer review

- Open access: articles freely available online

- High visibility within the field

- Retaining the copyright to your article

Submit your next manuscript at $\gg$ springeropen.com 